\title{
Quellen- und Literaturverzeichnis
}

\author{
Quellen
}

\author{
Institut für Stadtgeschichte, Frankfurt am Main
}

Bestände: Kulturamt, Historisches Museum (HiMu), Städtische Bühnen (SB), Protokolle des Magistrats, der Stadtverordnetenversammlung, des Kulturausschusses und der Theaterdeputation, Zeitungsausschnittsammlungen, personengeschichtliche Sammlungen, Wahlen, Nachlaß Rudi Arndt

\section{Landesarchiv Berlin}

Bestand Deutscher Städtetag (u.a. die Protokolle des Kulturpolitischen Ausschusses des DST)

Bibliothek des Deutschen Instituts für Urbanistik, Berlin

diverse auch graue Literatur

Archiv der sozialen Demokratie (Friedrich-Ebert-Stiftung), Bonn

Bestände: SPD-Unterbezirk Frankfurt am Main, SPD-Bezirk Hessen-Süd, Nachlaß Rudi Arndt

Archiv für Christlich-Demokratische Politik (Konrad-Adenauer-Stiftung), St. Augustin

Bestände: CDU-Kreisverband Frankfurt, CDU-Landesverband Hessen, Kommunalpolitische Vereinigung, Nachlässe Alfred Dregger und Wilhelm Fay, Depositum Walter Wallmann

\section{Archiv des Liberalismus (Friedrich-Naumann-Stiftung), Gummersbach}

FDP-Fachausschuß Kommunalpolitik, FDP-Landesverband Hessen, Nachlaß Wolfgang Mischnick

\section{Privatarchiv Ernst Gerhardt, Frankfurt am Main}

Faszikel „Gruppe 70“ 
Zeitungen und Zeitschriften

\section{Frankfurter Presse:}

Frankfurter Allgemeine Zeitung, Frankfurter Rundschau, Frankfurter Neue Presse, Abendpost/Nachtausgabe, Kulturjournal Frankfurt, Der Sozialdemokrat (Frankfurt)

Überregionale Zeitungen und Zeitschriften:

Süddeutsche Zeitung, Die Welt, Der Spiegel, Die Zeit, Rheinischer Merkur, Stuttgarter Zeitung; Der Städtetag, Archiv für Kommunalwissenschaften, Die demokratische Gemeinde, Kommunalpolitische Blätter, Das Rathaus. Zeitschrift für Kommunalpolitik; Museumskunde, Die deutsche Bühne, Theater heute, Volksbühnen-Spiegel 


\section{Literatur $^{1}$}

Abgeordnete des Deutschen Bundestages, Aufzeichnungen und Erinnerungen. Hg. vom Deutschen Bundestag, Bd.12 (Karl Becker), Boppard am Rhein 1993

Agnoli, Johannes/Brückner, Peter, Die Transformation der Demokratie, West-Berlin 1967

Ahlberg, René, Ursachen der Revolte. Analyse des studentischen Protestes, Stuttgart u.a. 1972

Aktionsgemeinschaft Westend (Hg.), Ende oder Wende? Studie zur Situation des Westends von Frankfurt am Main (unter Mitarb. von Odina Bott), Steinbach/Ts. 1969

Albrecht, Willy, Der Sozialistische Deutsche Studentenbund (SDS). Vom parteikonformen Studentenverband zum Repräsentanten der neuen Linken, Bonn 1994

Albrecht, Willy, „,Unter den Talaren ...': Studentenbewegung und Sozialdemokratie bis 1968“, in: Dowe, Dieter (Hg.), Partei und Soziale Bewegung. Kritische Beiträge zur Entwicklung der SPD seit 1945, Bonn 1993, S. 59-80

Aly, Götz, Unser Kampf: 1968 - ein irritierter Blick zurück, Bonn 2008

Amlung, Ullrich (Hg.), „Leben ist immer ein Anfang!“ Erwin Piscator 1893-1966. Der Regisseur des politischen Theaters, Marburg 1993

Appel, Rudolf Heinrich, Heißer Boden. Stadtentwicklung und Wohnprobleme in Frankfurt am Main (Eine Publikation des Presse- und Informationsamtes der Stadt Frankfurt am Main), Frankfurt a.M. 1974

Arbeiterjugendbewegung in Frankfurt 1904-1945. Material zu einer verschütteten Kulturgeschichte, Frankfurt 1978 (Ausstellungskatalog. Organisation: Detlef Hoffmann, Doris Pokorny, Albrecht Werner)

Arend, Peter, Die innerparteiliche Entwicklung der SPD 1966-1975, Bonn 1975

Auer, Frank von, Alfred Dregger. Ein kritisches politisches Porträt, Berlin 1974

Baier, Lothar u.a. (Hg.), Die Früchte der Revolte. Über die Veränderung der politischen Kultur durch die Studentenbewegung, Berlin 1988

Balcar, Jaromír, Politik auf dem Lande: Studien zur bayerischen Provinz 1945 bis 1972, München 2004

Balser, Frolinde, Die Anfänge der Erwachsenenbildung in Deutschland in der ersten Hälfte des 19. Jahrhunderts. Eine kultursoziologische Deutung, Stuttgart 1959

Balser, Frolinde, Sozial-Demokratie 1848/49-1863. Die erste deutsche Arbeiterorganisation ,Allgemeine deutsche Arbeiterverbrüderung' nach der Revolution, Stuttgart 1962

Balser, Frolinde, Aus Trümmern zu einem europäischen Zentrum - Geschichte der Stadt Frankfurt am Main 1945-1989, Frankfurt a. M. 1995

Balzer, Friedrich-Martin/Bock, Hans Manfred/Schöler, Uli, Wolfgang Abendroth - Wissenschaftlicher Politiker. Bio-bibliographische Beiträge, Opladen 2001

Bamberg, Ulrich u. a., Aber ob die Karten voll ausgereizt sind ... 10 Jahre Mitbestimmungsgesetz 1976 in der Bilanz, Bonn 1987

Baring, Arnulf, in Zusammenarbeit mit Manfred Görtemaker, Machtwechsel. Die Ära Brandt/Scheel, 2. Aufl., Stuttgart 1982

Bausch, Ulrich M., Die Kulturpolitik der US-amerikanischen Information Control Division in Württemberg-Baden von 1945-1949, Stuttgart 1992

Becker, Jörg, Rudi Arndt. Frankfurts neuer OB. Eine kritische Analyse von Jörg Becker, Frankfurt 1972

Becker, Hartmuth/Dirsch, Felix/Winckler, Stefan, Die 68er und ihre Gegner. Der Widerstand gegen die Kulturrevolution, Graz/Stuttgart 2003

Beer, Rüdiger R., Schwerpunkte kommunaler Kulturpolitik. Europäische Vergleiche, Stuttgart u.a. 1968

Behrens, Roger, Kritische Theorie, Hamburg 2002

1 Bei Aufsätzen in Sammelbänden, aus denen mehrere Beiträge zitiert werden, wird aus Platzgründen nur ein Kurztitel des Sammelbandes angegeben. Dessen vollständiger Titel ist am entsprechenden Ort nur einmal in Gänze genannt. 
Benz, Wolfgang (Hg.), Die Geschichte der Bundesrepublik Deutschland, Bd.3: Gesellschaft, Bd. 4: Kultur, Frankfurt a. M. 1989

Berkemeier, Karl H., Das kommunale Scheinparlament. Ausgeschaltet aus dem Planungsprozeß. Bilanz eines Stadtverordneten, in: Zeitschrift für Parlamentsfragen 3 (1972), S. 202-208

Berliner Ensemble (Hg.), Theaterarbeit: 6 Aufführungen des Berliner Ensembles, Dresden 1952

Berlit, Anna Christina, Notstandskampagne und Rote-Punkt-Aktion. Die Studentenbewegung in Hannover 1967-1969, Bielefeld 2007

Bermbach, Udo, Probleme des Parteienstaates - Der Fall Littmann, in: Zeitschrift für Parlamentsfragen, Heft 3, 1970, S.342-363

Bermbach, Udo, Repräsentation, imperatives Mandat und Recall. Zur Frage der Demokratisierung im Parteienstaat, in: Theory and politics. Festschrift zum 70. Geburtstag für Carl Joachim Friedrich, Haag 1971, S. 497-525

Bernhard, Patrick, Zivildienst zwischen Reform und Revolte. Eine bundesdeutsche Institution im gesellschaftlichen Wandel 1961-1982, München 2005

Bethmann, Johann-Philipp Freiherr von, Bankiers sind auch Menschen. 225 Jahre Bankhaus Gebrüder Bethmann, Frankfurt a. M. 1973

Beye, Peter, Aufgaben des Museums in der Gegenwart, in: Bott, Das Museum, S. 17-20

Bieling, Rainer, Die Tränen der Revolution. Die 68er zwanzig Jahre danach, Berlin 1988

Biermann, Alfons W. (Hg. im Auftrag des Rheinischen Museumsamtes), Vom Elfenbeinturm zur Fußgängerzone: Drei Jahrzehnte deutsche Museumsentwicklung. Versuch einer Bilanz und Standortbestimmung, Opladen 1996

Bollinger, Stefan, 1968 - die unverstandene Weichenstellung, Berlin 2008

Bondy, Luc, Wo war ich? Einbildungen, Frankfurt a. M. 2000

Börnsen, Gert, Innerparteiliche Opposition. Jungsozialisten und SPD, Hamburg 1969

Bösch, Frank, Die Adenauer-CDU. Gründung, Aufstieg und Krise einer Erfolgspartei 1945-1969, München/Stuttgart 2001

Bösch, Frank, Die Krise als Chance. Die Neuformierung der Christdemokraten in den siebziger Jahren, in: Jarausch, Das Ende der Zuversicht, S. 296-312

Boom, Pierre, Der fremde Vater. Der Sohn des Kanzlerspions Guillaume erinnert sich, Berlin 2005

Bott, Gerhard (Hg.), Das Museum der Zukunft. 43 Beiträge zur Diskussion über die Zukunft des Museums, Köln 1970

Braun, Karlheinz, 1959 und 1969 von 1999 aus gesehen, in: Verleger als Beruf. Siegfried Unseld zum fünfundsiebzigsten Geburtstag, Frankfurt a. M. 1999, S. 28-34

Braunthal, Gerard, Politische Loyalität und Offentlicher Dienst. Der „Radikalenerlaß“ von 1972 und die Folgen, Marburg 1992

Bracher, Karl Dietrich, Betrachtungen zum Problem der Macht, Opladen 1991

Brecht, Bertolt, „Werke“. Große kommentierte Berliner und Frankfurter Ausgabe. Hg. von Hecht, Werner/Knopf, Jan/Mittenzwei, Werner/Müller, Klaus-Detlef, Bd.2: „Stücke 2“, Berlin u.a. 1988

Bremerich-Vos, Albert (Hg.), Handlungsfeld Deutschunterricht im Kontext. Festschrift für Hubert Ivo, Frankfurt a. M. 1993

Briem, Jürgen, Der SDS. Die Geschichte des bedeutendsten Studentenverbandes der BRD seit 1945, Frankfurt a.M. 1976

Brook, Peter, Der leere Raum. Möglichkeiten des heutigen Theaters, Hamburg 1969

Buckwitz, Harry, Theater für alle. Theater ohne Krise. Ein Vortrag. Gehalten auf dem 16. Volksbühnentag in Stuttgart am 12. Juni 1954, Berlin/Grunewald 1954 (Senatsbibliothek Berlin)

Buckwitz, Harry, Harry Buckwitz, Schauspieler, Regisseur, Intendant: 1904-1987. „den lieb’ ich, der Unmögliches begehrt", Berlin 1998

Bude, Heinz, Das Altern einer Generation. Die Jahrgänge 1938-1948, Frankfurt a. M. 1995

Burckhardt, Jacob, Weltgeschichtliche Betrachtungen. Über geschichtliches Studium, Darmstadt 1956 (zuerst 1905)

Busche, Jürgen, Die 68er. Biographie einer Generation, Berlin 2003 
Büscher, Barbara, Wirklichkeitstheater, Straßentheater, Freies Theater: Entstehung und Entwicklung freier Gruppen in der Bundesrepublik Deutschland 1968-76, Frankfurt a.M. u. a. 1987

Butterwege, Christoph, Jungsozialisten und SPD. Die Widerspiegelung sozioökonomischer Entwicklungstendenzen im Verhältnis des sozialdemokratischen Jugendverbandes zu seiner „Mutterpartei“, Hamburg 1975

Carstensen, Uwe B., Klaus Michael Grüber, Frankfurt a. M. 1988

CDU-Landesverband Hessen (Hg.), Marx statt Rechtschreibung. Rahmenrichtlinien in Hessen. Gesellschaftslehre und Deutsch, Wiesbaden 1973

Chaussy, Ulrich, Die drei Leben des Rudi Dutschke. Eine Biographie, Berlin 1993

Christ, Hannelore/Holzschuh, Horst/Merkelbach, Valentin/Raitz, Walter/Stückrath, Jörn, Hessische Rahmenrichtlinien Deutsch. Analyse und Dokumentation eines bildungspolitischen Konflikts, Düsseldorf 1974

Christ, Hannelore u. a., Theaterstücke zum Radikalenerlaß. Texte, Bilder und Dokumente. Bearbeitet und zusammengestellt vom Arbeitskreis Theater Frankfurt. Mit einem Vorwort von Wolf-Dieter Narr, Offenbach 1978

Chromik, Johanna Elisabeth, Kulturpolitik in der Bundesrepublik Deutschland von 1960 bis 1973. Theaterpolitik in Frankfurt am Main, unveröff. Magisterarbeit am Institut für Kulturwissenschaften der Universität Leipzig 2004 (Bibliothek des IfSG)

Claussen, Detlev (Hg.), Spuren der Befreiung - Herbert Marcuse. Ein Materialbuch zur Einführung in sein politisches Denken, Darmstadt 1981

Claussen, Detlev, Theodor W. Adorno. Ein letztes Genie, Frankfurt a. M. 2003

Clemens, Gabriele B., Britische Kulturpolitik in Deutschland 1945-1949: Literatur, Film, Musik und Theater, Stuttgart 1997

Dahl, Robert A., Who governs? Democracy and Power in an American City, New Haven u.a. 1961

Dahlke, Matthias, „Nur eingeschränkte Krisenbereitschaft“. Die staatliche Reaktion auf die Entführung des CDU-Politikers Peter Lorenz 1975, in: VfZ 55 (2007), S.641-678

Dahrendorf, Ralf, Für eine Erneuerung der Demokratie in der Bundesrepublik. Sieben Reden und andere Beiträge zur deutschen Politik 1967-1968, München 1968

Daiber, Hans, Deutsches Theater seit 1945. Bundesrepublik Deutschland, Deutsche Demokratische Republik, Österreich, Schweiz, Stuttgart 1976

Decker, Karl-Viktor, Ein Museumsmann alter Schule verabschiedet sich, in: Jung, „Zum Sehen geboren, zum Schauen bestellt", S. 25-28

Degen, Tassilo, Der Tendenzbetrieb im Betriebsverfassungsrecht. Die Problematik des $₫ 81$ Betriebsverfassungsgesetz im Licht der neueren Rechtsentwicklung, Würzburg 1969

Demm, Sabine, Die Studentenbewegung von 1968 in Frankfurt am Main. Eine Chronologie, in: Archiv für Frankfurts Geschichte und Kunst 67 (2001), S. 161-247

Der deutsche Adler. Funktionen eines politischen Symbols. Katalog einer Ausstellung des Frankfurter Kunstvereins im Steinernen Haus am Römerberg, 29. Juni-12. August 1973, Bearbeiter: Reiner Diederich und Richard Grübling, Frankfurt 1973

Der gesellschaftliche Ort der ,68er` Bewegung, 17 Beiträge, in: Westfälische Forschungen 48 (1998) S. 1-357

Der Verlust der Mitte: Orientierungslosigkeit nach 25 Jahren Kulturrevolution; Studientagung der Evangelischen Notgemeinschaft Deutschland e.V. vom 15.-17. Oktober 1993 in Hannover, Weßling 1994 (Erneuerung und Abwehr / Beiheft; 57)

Derlien, Hans-Ulrich/Gürtler, Christoph/Holler, Wolfgang/Schreiner, Hermann Josef, Kommunalverfassung und kommunales Entscheidungssystem. Eine vergleichende Untersuchung in vier Gemeinden, Meisenheim am Glan 1976

Deutsche Forschungsgemeinschaft, Die Notlage der Museen in der Bundesrepublik Deutschland. Appell zur Soforthilfe, Bonn - Bad Godesberg 1971

Deutscher Städtetag (Hg.), Wege zur menschlichen Stadt. Vorträge, Aussprachen und Ergebnisse der 17. Hauptversammlung des Deutschen Städtetages vom 2. bis 4. Mai 1973 in Dortmund, Köln 1973 
Deutscher Städtetag (Hg.), Kultur in den Städten. Eine Bestandsaufnahme, Stuttgart u.a. 1979

Die Zukunft beginnt in der Vergangenheit. Museumsgeschichte und Geschichtsmuseum, Schriften des Historischen Museums Frankfurt a.M., Festschrift für Hans Stubenvoll, Gießen 1982

Diers, Andreas, Vom Rätegedanken zur Formel des „demokratischen und sozialen Rechtsstaats“. Ein Leben in der Arbeiterbewegung - Wolfgang Abendroth (1906-1985), in: Die Linkspartei. PDS Bremen (Hg.), Der Partisanenprofessor im Land der Mitläufer. Wolfgang Abendroth zum Hundertsten Geburtstag, Bremen 2006, S. 8-15

Dinné, Olaf/Grünwald, Jochen/Kuckuk, Per (Hg.), anno dunnemals - 68 in Bremen, Bremen 1998

Ditges, Peter, Kommunale Kulturarbeit: System und Beispiele, Stuttgart u.a. 1985

Ditges, Peter, Rechtsprobleme kommunaler Kulturarbeit, Würzburg 1986

Doering-Manteuffel, Anselm, Nach dem Boom. Brüche und Kontinuitäten der Industriemoderne seit 1970, in: VfZ 55 (2007), S. 559-581

Doering-Manteuffel, Anselm/Raphael, Lutz, Nach dem Boom. Perspektiven auf die Zeitgeschichte seit 1970, Göttingen 2008

Dorst, Tankred, Was ich Peter Palitzsch verdanke, in: Iden, Peter Palitzsch, S. 173-178

Dostal, Caroline, 1968 - Demonstranten vor Gericht. Ein Beitrag zur Justizgeschichte der Bundesrepublik, Frankfurt a.M. 2006

Dregger, Alfred, Systemveränderung. Brauchen wir eine andere Republik?, Stuttgart 1972

Dünnwald, Rolf, Die Rechtsstellung des Theaterintendanten, Univ. Diss. Köln 1964

Düring, Sonja, Sexuelle Befreiung, in: Landgrebe/Plath, '68 und die Folgen, S. 109-112

Dutschke, Rudi, Mein langer Marsch. Reden, Schriften und Tagebücher aus 20 Jahren, Reinbek bei Hamburg 1980

Duve, Freimut (zusammengestellt von), Der Thesenstreit um „Stamokap“. Die Dokumente zur Grundsatzdiskussion der Jungsozialisten, Reinbek bei Hamburg 1973

Eckert, Rainer, Das ist „demokratischer Sozialismus“. Eine Antwort auf Johano Strassers Frage: „Was ist demokratischer Sozialismus?“, Frankfurt a.M. 1975

Eckert, Rainer, Die Krise der SPD, Frankfurt 1982

Ehmsen, Stefanie, Der Marsch der Frauenbewegung durch die Institutionen. Die Vereinigten Staaten und die Bundesrepublik im Vergleich, Münster 2008

Ellwein, Thomas, Krisen und Reformen - Die Bundesrepublik seit den sechziger Jahren, München 1989

Engelmann, Bernt, Das Reich zerfiel, die Reichen blieben. Deutschlands Geld- und Machtelite. Mit Rangliste der 500 großen alten Vermögen, Hamburg 1972

Engelmann, Bernt, Wir Untertanen. Ein deutsches Anti-Geschichtsbuch, München u. a. 1974

Faulenbach, Bernd, Die Siebzigerjahre - ein sozialdemokratisches Jahrzehnt, in: Archiv für Sozialgeschichte 44 (2004), S.1-37

Faulstich, Werner (Hg.), Die Kultur der 60er Jahre, München 2003

Faulstich, Werner (Hg.), Die Kultur der 70er Jahre, München 2004

Felsch, Margot, Aus der Chef-Etage des Römers. Begegnungen mit Walter Kolb, Werner Bockelmann, Willi Brundert, Walter Möller, Rudi Arndt und Walter Wallmann, Frankfurt a.M. 1981

Fichter, Tilman, SDS und SPD. Parteilichkeit jenseits der Partei, Opladen 1988

Fichter, Tilman/Lönnendonker, Siegward, Kleine Geschichte des SDS. Der Sozialistische Deutsche Studentenbund von Helmut Schmidt bis Rudi Dutschke, 4. überarb. u. erg. Aufl., Essen 2007

Flechtheim, Ossip K., Der Marsch der DKP durch die Institutionen. Sowjetmarxistische Einflußstrategien und Ideologien, Frankfurt 1981

Fogt, Helmut, Politische Generationen. Empirische Bedeutung und theoretisches Modell, München 1980

Frank, Rainer, Kultur auf dem Prüfstand. Ein Streifzug durch 40 Jahre kommunaler Kulturpolitik, München 1990 
Frank, Rainer, Von der Opernbühne zum soziokulturellen Stadtteilzentrum. Zur Kulturpolitik in der Kommune - Analyse ihrer Entwicklung in der Bundesrepublik und Bewertung aktueller kommunaler Kulturarbeit, Konstanz 1987

Frankfurter Biographie. Personengeschichtliches Lexikon. Im Auftrag der Frankfurter Historischen Kommission. Klötzer, Wolfgang (Hg.), Erster Band (A-L), Frankfurt a.M. 1994, Zweiter Band (M-Z), Frankfurt a.M. 1996

Frei, Norbert, 1968. Jugendrevolte und globaler Protest, Bonn 2008 (Lizenzausgabe für die Bundeszentrale für politische Bildung)

Frese, Matthias/Paulus, Julia/Teppe, Karl, Demokratisierung und gesellschaftlicher Aufbruch. Die sechziger Jahre als Wendezeit der Bundesrepublik, Paderborn u. a. 2003

Frey, Erich, Der Tendenzbetrieb im Recht der Betriebsverfassung und des Arbeitsverhältnisses, Heidelberg 1959

Frey, Erich, Der Tendenzschutz im BetrVG 1972, Diss. Heidelberg 1974

Frey, Rainer/Holler, Wolfgang, Die Grundtypen der Gemeindeverfassung in der Bundesrepublik, in: Frey, Rainer (Hg.), Kommunale Demokratie. Beiträge für die Praxis der kommunalen Selbstverwaltung, Bonn-Bad Godesberg 1976, S. 241-274

Friedeburg, Ludwig von, Bildungsreform in Deutschland. Geschichte und gesellschaftlicher Widerspruch, Frankfurt a. M. 1989

Friedrich, Regine, Mündel will Vormund sein! Oder: Die „TAT-Story“, in: Bleibohm, Katharina/Sprang, Wolfgang (Hg.), Neue Szene Frankfurt 1970-76, Frankfurt a.M. 1976, S.22-27

Frisch, Peter, Extremistenbeschluß. Zur Frage der Beschäftigung von Extremisten im öffentlichen Dienst mit grundsätzlichen Erläuterungen, Argumentationskatalog, Darstellung extremistischer Gruppen und einer Sammlung einschlägiger Vorschriften, Urteile und Stellungnahmen, 4. überarb. u. aktual. Aufl., Leverkusen 1977

Fröhlich, Michael, Imperialismus. Deutsche Kolonial- und Weltpolitik 1880-1914, 2. Aufl. München 1997

Fünf Jahrzehnte kommunale Kulturpolitik, hg. vom Deutschen Städtetag (bearb. von Helmut Lange), Köln 1992

Gabriel, Oscar W., Strukturen politischer Willensbildung in der Gemeinde, in: ders. (Hg.), Kommunalpolitik im Wandel der Gesellschaft. Eine Einführung in die Probleme der Willensbildung in der Gemeinde, Mainz 1979, S. 63-93

Gall, Lothar, Der Bankier. Hermann Josef Abs. Eine Biographie, München 2004

Gau, Doris, Kultur als Politik. Eine Analyse der Entscheidungsprämissen und des Entscheidungsverhaltens in der kommunalen Kulturpolitik, München 1990

Gebauer, Annekatrin, Der Richtungsstreit in der SPD. Seeheimer Kreis und Neue Linke im innerparteilichen Machtkampf, Wiesbaden 2005

Gehlen, Arnold, Urmensch und Spätkultur. Philosophische Ergebnisse und Aussagen, Bonn 1956

Geiss, Imanuel, Zum Streit ums Historische Museum in Frankfurt, in: Hoffmann/Junker/ Schirmbeck, Geschichte als öffentliches Ärgernis, S.7-14

Geiss, Imanuel/Ulrich,Volker (Hg.), Fünfzehn Millionen beleidigte Deutsche oder Woher kommt die CDU. Beiträge zur Kontinuität der bürgerlicher Parteien, Reinbek bei Hamburg 1972

Gilcher-Holtey, Ingrid, Die Phantasie an die Macht. Mai 68 in Frankreich, 2. Aufl., Frankfurt a. M. 2001 (zuerst 1995)

Gilcher-Holtey, Ingrid (Hg.), 1968. Vom Ereignis zum Gegenstand der Geschichtswissenschaft, Göttingen 2008 (zuerst 1998)

Gilcher-Holtey, Ingrid, Die 68er Bewegung. Deutschland - Westeuropa - USA, München 2001

Gilcher-Holtey, Ingrid/Kraus, Dorothea/Schößler, Franziska (Hg.), Politisches Theater nach 1968. Regie, Dramatik und Organisation, Frankfurt a.M./New York 2006

Glaser, Hermann, Bemerkungen zum Begriff der Kulturrevolution, in: Tribüne 8 (1969), S. 3348-3366

Glaser, Hermann, Kulturgeschichte der Bundesrepublik, Bd.2: Zwischen Grundgesetz und Großer Koalition 1949-1976, München u.a. 1986, Bd.3: Zwischen Protest und Anpassung 1968-1989, München u.a. 1989 
Glaser, Hermann, Bildungs- und Kulturpolitik. Von der Reeducation zum Schönerleben, in: Hoffmann, Hilmar/Klotz, Heinrich (Hg.), Die Kultur unseres Jahrhunderts. 1945-1960, Düsseldorf/Wien/New York 1991, S. 27-43

Gniffke, Kai, Volksbildung in Frankfurt am Main 1890-1990. Festschrift zum 100jährigen Jubiläum, Frankfurt a.M. 1990

Görtemaker, Manfred, Geschichte der Bundesrepublik Deutschland. Von der Gründung bis zur Gegenwart, München 1999

Grauhahn, Rolf-Richard, Einführung: Lokale Politikforschung, in: ders. (Hg.), Lokale Politikforschung, Bd.1, Frankfurt a. M. 1975, S.11-37

Grauhan, Rolf-Richard, Der politische Willensbildungsprozeß in der Großstadt, in: Großstadt-Politik. Texte zur Analyse und Kritik lokaler Demokratie, hg. von Rolf-Richard Grauhan, Gütersloh 1972, S.145-162

Grauhan, Rolf-Richard, Großstadtpolitik, Gütersloh 1972

Greifenhagen, Martin/Scheer, Hermann (Hg.), Die Gegenreform. Zur Frage der Reformierbarkeit von Staat und Gesellschaft, Reinbek bei Hamburg 1975

Grimm, Thomas, Linke Vaterlandsgesellen. Sozialisten, Anarchisten, Kommunisten, Raufbolde und andere Unangepasste, Berlin 2003

Gröteke, Iris, Gegen den Strich gebürstet? Anspruch und Selbstverständnis, in: Kritische Berichte 2/1999 (Thema: 30 Jahre Ulmer Verein), S.7-15

Grossarth-Maticek, Ronald, Radikalismus. Untersuchungen zur Persönlichkeitsentwicklung westdeutscher Studenten, Basel 1979

Grossarth-Maticek, Ronald, Revolution der Gestörten? Motivationsstrukturen, Ideologien und Konflikte bei politisch engagierten Studenten, Heidelberg 1975

Guggenberger, Bernd, Utopische Freiheit. Rätedemokratie und imperatives Mandat, Köln 1974

Guggenberger, Bernd, Wohin treibt die Protestbewegung? Junge Rebellen zwischen Subkultur und Parteikommunismus. Ursachen und Folgen der Unfähigkeit zur Politik, Freiburg u.a. 1975

Guillaume, Günter, Die Aussage. Wie es wirklich war, Frankfurt a.M. u.a. 1993

Haasis, Hans-Arthur, Kommunalpolitik und Machtstruktur. Eine Sekundäranalyse deutscher empirischer Gemeindestudien, Frankfurt a. M. 1978

Häberle, Peter, Das Kulturverfassungsrecht der Bundesrepublik Deutschland, in: APuZ 28 (1985), S. 11-30

Häberle, Peter, Kulturpolitik in der Stadt - ein Verfassungsauftrag, Karlsruhe u.a. 1979

Hammer-Schenk, Harold/Waskönig, Dagmar/Weiss, G., Kunstgeschichte gegen den Strich gebürstet? 10 Jahre Ulmer Verein, 1968-1978. Geschichte in Dokumenten (Neuaufl. der Ausgabe von 1979; neu bearb. von Iris Grötecke), Marburg 1997

Handbuch zur Statistik der Parlamente und Parteien in den westlichen Besatzungszonen und in der Bundesrepublik Deutschland. Teilband II. CDU und CSU. Mitgliedschaften und Sozialstruktur 1945-1990, bearb. von Corinna Franz und Oliver Gnad, Düsseldorf 2005

Handbuch zur Statistik der Parlamente und Parteien in den westlichen Besatzungszonen und in der Bundesrepublik Deutschland. Teilband IV. SPD, KPD und kleinere Parteien des linken Spektrums sowie DIE GRÜNEN, bearb. von Josef Boyer und Till Kössler, Düsseldorf 2005

Hansert, Andreas, Bürgerkultur und Kulturpolitik in Frankfurt am Main. Eine historischsoziologische Rekonstruktion, Frankfurt a. M. 1992

Harbeck, Karl-Heinz, Das „Imperative Mandat“ - eine falsche Antwort auf eine richtige Frage, Kiel 1974 (Amt für staatsbürgerliche Bildung in Schleswig-Holstein)

Heidenreich, Bernd/Wolf, Werner (Hg.), Der Weg zur stärksten Partei 1945-1995: 50 Jahre CDU Hessen, Köln 1995

Heider, Ulrike, Keine Ruhe nach dem Sturm, Hamburg 2001

Heigl, Richard, Oppositionspolitik. Wolfgang Abendroth und die Entstehung der neuen Linken (1950-1968), Hamburg 2008

Hein, Bastian, Die Westdeutschen und die Dritte Welt. Entwicklungspolitik und Entwicklungsdienste zwischen Reform und Revolte 1959-1974, München 2006 
Hein, Dieter, Zwischen liberaler Milieupartei und nationaler Sammlungsbewegung. Gründung, Entwicklung und Struktur der Freien Demokratischen Partei 1945-1949, Düsseldorf 1985

Heinemann, Karl-Heinz/Jaitner, Thomas, Ein langer Marsch. 1968 und die Folgen, Köln 1993

Heinrichs, Werner, Kommunales Kulturmanagement. Rahmenbedingungen, Praxisfelder,

Managementmethoden, Baden-Baden 1999

Heinz-Jung-Stiftung (Hg.), Linke im Kalten Krieg. Autobiographische Berichte aus Frankfurt am Main 1945 bis 1968 (Red. David Salomon), Köln 2007

Held, Jutta, Konzeptionen historischer Museen, in: Kuhn/Schneider, Geschichte lernen im Museum, S.11-31

Hennis, Wilhelm, Demokratisierung. Zur Problematik eines Begriffs, in: Lehmbruch, Gerhard/von Beyme, Klaus/Fetscher, Iring (Hg.), Demokratisches System und politische Praxis der Bundesrepublik, München 1971, S.68-96

Hensel, Georg, Das Theater der siebziger Jahre. Kommentar, Kritik, Polemik, 2. Aufl., Stuttgart 1981

Herbert, Ulrich, Liberalisierung als Lernprozeß. Die Bundesrepublik in der deutschen Geschichte, in: ders. (Hg.), Wandlungsprozesse in Westdeutschland. Belastung, Integration, Liberalisierung 1945-1989, Göttingen 2002, S.7-49

Herlemann, Falko, Krise war immer. Von der Reform zur Resignation, in: Kritische Berichte 2/1999 (Thema: 30 Jahre Ulmer Verein), S. 16-25

Hermand, Jost, Die Kultur der Bundesrepublik Deutschland 1965-1985, München 1988

Hermann, Lutz, Georg Leber. Vom Maurer zum Staatsmann. Ein Porträt, Freudenstadt 1969

Hesselbach, Walter/Schmidt, Helmut/Oertzen, Peter von, Kämpfer ohne Pathos. Festschrift für Hans Matthöfer zum 60. Geburtstag am 25. September 1985, Bonn 1985

Hessische Gemeindeordnung (HGO) - Textausgabe -, hg. vom Hessischen Städtetag, Sonderdruck Nr.12, April 1985

Hildebrand, Klaus, Von Erhard zur Großen Koalition, 1963-1969 (Geschichte der Bundesrepublik Deutschland, Bd.4), Stuttgart 1984

Hochkeppel, Willy, Die Rolle der Neuen Linken in der Kulturindustrie, München 1972

Hockerts, Hans Günter, Parteien in Bewegung. Über die Flexibilität des Parteiensystems in der Reformära der Bonner Republik (1966-1974), in: Hildebrand, Klaus/Wengst, Udo/ Wirsching, Andreas (Hg.), Geschichtswissenschaft und Zeiterkenntnis. Von der Aufklärung bis zur Gegenwart. Festschrift zum 65. Geburtstag von Horst Möller, München 2008, S. 225-240

Hockerts, Hans Günter, Das Profil der Reformära, in: Bundesministerium für Arbeit und Soziales und Bundesarchiv (Hg.), Geschichte der Sozialpolitik in Deutschland seit 1945, Bd.5: Bundesrepublik Deutschland 1966-1974. Eine Zeit vielfältigen Aufbruchs, BadenBaden 2006, S.3-155

Hodenberg, Christina von/Siegfried, Detlef, Wo „1968“ liegt. Reform und Revolte in der Geschichte der Bundesrepublik, Göttingen 2006

Hodenberg, Christina von/Siegfried, Detlef, Reform und Revolte. 1968 und die langen sechziger Jahre in der Geschichte der Bundesrepublik, in: dies., Wo „1968“ liegt, S.7-14

Hodenberg, Christina von, Der Kampf um die Redaktionen. „1968“ und der Wandel der westdeutschen Massenmedien, in: dies./Siegfried, Wo „1968“ liegt, S.139-163

Hoffmann, Detlef, „Laßt Objekte sprechen!“. Bemerkungen zu einem verhängnisvollen Irrtum, in: Spickernagel/Walbe (Hg.), Das Museum, S.101-120

Hoffmann, Detlef, Drei Jahrzehnte Museumsentwicklung in der Bundesrepublik - Trends, Strukturen, Perspektiven, in: Biermann, Vom Elfenbeinturm zur Fußgängerzone, S.13-23

Hoffmann, Detlef, Die Karlsfresken Alfred Rethels, Freiburg 1968

Hoffmann, Detlef/Junker, Almut/Schirmbeck, Peter (Hg.), Geschichte als öffentliches Ärgernis oder ein Museum für die demokratische Gesellschaft. Das historische Museum in Frankfurt am Main und der Streit um seine Konzeption, Fernwald 1974

Hoffmann, Detlef, Ein demokratisches Museum (I): Geschichte und Konzeption, in: Hoffmann/Junker/Schirmbeck, Geschichte als öffentliches Ärgernis, S. 15-24 
Hoffmann, Detlef, Geschichte - keine Geschichtchen. Erfahrungen am Historischen $\mathrm{Mu}-$ seum Frankfurt a. M., in: Vorgänge 26 (1977), S. 92-97

Hoffmann, Hilmar, Tauben, reisende Boten. Kulturgeschichte und Sport der Brieftaube, Duisburg 1963

Hoffmann, Hilmar, 1 Jahr Kulturarbeit in Frankfurt. Ein Rechenschaftsbericht, Presse- und Informationsamt der Stadt Frankfurt am Main (Hg.), o. O. 1972

Hoffmann, Hilmar, Kultur für alle. Perspektiven und Modelle, aktual. u. erw. Ausgabe Frankfurt a.M. 1981

Hoffmann, Hilmar, Ihr naht Euch wieder, schwankende Gestalten. Erinnerungen, Hamburg 1999

Hoffmann, Hilmar (Hg.), Perspektiven der kommunalen Kulturpolitik - Beschreibungen und Entwürfe, Frankfurt 1974

Hoffmann, Hilmar, Theater als organischer Teil der Gesellschaft, in: Loschütz, War das was?, S. 11-16

Hoffmann, Hilmar/Klotz, Heinrich (Hg.), Die Kultur unseres Jahrhunderts, Bd.5: Die Sechziger, Bd.6: 1970-1990, Düsseldorf/Wien/New York/Moskau 1987 bzw. 1990

Hoffmann, Hilmar, Kulturpolitik - Auf dem Weg zur Kulturgesellschaft, in: Hoffmann/ Klotz, Die Kultur unseres Jahrhunderts, Bd. 6: 1970-1990, S. 33-53

Hoffmann, Ludwig (Hg. unter Mitarbeit von Klaus Pfützner), Theater der Kollektive. Proletarisch-revolutionäres Berufstheater in Deutschland 1928-1933. Stücke, Dokumente, Studien, 2 Bde., Berlin 1980

Hollenstein, Erich, Kulturelles bargaining. Ein Aspekt alternativer soziokultureller Aktivität, Essen 1978

Holler, Wolfgang, Ziele und Rahmenbedingungen kommunaler Politik. Eine vergleichende Analyse der kommunalpolitischen Grundsatzprogramme von SPD, CDU/CSU und FDP, in: Naßmacher, Karl-Heinz (Hg.), Kommunalpolitik und Sozialdemokratie. Der Beitrag des demokratischen Sozialismus zur kommunalen Selbstverwaltung, Bonn-Bad Godesberg 1977, S. 127-140

Horn, Wolfgang, Kulturpolitik in Düsseldorf. Situation und Neubeginn nach 1945, Opladen 1981

Hubert, Ivo/Merkelbach, Valentin, Abschied vom klassischen Schulfach. Zum Beispiel Deutsch. Bearb. Dokumentation vom Schulbuchkongreß der Sozialdemokratischen Wählerinitiative Bonn, „Muß Schule dumm machen?“, Heidelberg 1972

Hübner, Kurt/Schmidt, Dietmar N./Hübner, Kurt, Von der Leidenschaft eines Theatermenschen, Leipzig 2006

Hug, Markus, Kultur- und Freizeitpolitik in der Mittelstadt. Zum Beispiel Universitätsstadt Tübingen, Tübingen 1986

Iden, Peter, Die Schaubühne am Halleschen Ufer, 1970-1979, München u. a. 1979

Iden, Peter, „Der Schein, was ist er, dem das Wesen fehlt?“ Zur Entwicklung des Schauspieltheaters in Frankfurt; Vortrag gehalten auf Einladung des Patronatsvereines für die Theater der Stadt Frankfurt am Main, 28. September 1992, im Nachtfoyer des Schauspielhauses (Bibliothek des IfSG)

Iden, Peter, Peter Palitzsch. Theater muß die Welt verändern, Berlin 2005

Initiative für die Freiheit der Theaterarbeit (Hg.), Der Freiheit eine Gasse. Dokumentation zur Zensur im Theater, Offenbach 1978

Institut für angewandte Sozialwissenschaft, Infas-Report Wahlen Bayern und Hessen, Analysen und Dokumente zu den Landtagswahlen 1974, Bonn - Bad Godesberg 1974

Ismayr, Wolfgang, Das politische Theater in Westdeutschland, 2. Aufl., Königstein/Ts. 1985

Jäger, Lorenz, Adorno. Eine politische Biographie, München 2003

Jahn, Hans Edgar (Hg.), CDU und Mitbestimmung. Der Weg zur Mitbestimmungsformel der CDU auf dem Parteitag 1968, Stuttgart 1969

Jarausch, Konrad H. (Hg.), Das Ende der Zuversicht. Die siebziger Jahre als Geschichte?, Göttingen 2008

Jarausch, Konrad H., Die Umkehr. Deutsche Wandlungen 1945-1995, Bonn 2004 
Jesse, Eckhard, Biographisches Porträt. Horst Mahler, in: Jahrbuch Extremismus \& Demokratie 13 (2001), S. 183-199

Johler, Jens, Der Falsche, Ungekürzte Ausgabe, München 1997

Jonas, Friedrich, Die Institutionenlehre Arnold Gehlens, Tübingen 1966

Juchler, Ingo, Rebellische Subjektivität und Internationalismus: Der Einfluß Herbert Marcuses und der nationalen Befreiungsbewegungen in der sog. Dritten Welt auf die Studentenbewegung in der BRD, Marburg 1989

Junker, Almut, Historische Dokumentation 9.-15. Jahrhundert, in: Hoffmann/Junker/ Schirmbeck, Geschichte als öffentliches Ärgernis, S. 27-68

Jureit, Ulrike, Generationenforschung, Göttingen 2006

Jürgs, Michael, Günter Grass. Eine deutsche Biografie, erw. Neuausgabe, München 2007

Jung, Sabine (Hg.), „Zum Sehen geboren, zum Schauen bestellt“: Faust II, 5. Akt, Tiefe Nacht. Horst Reber zum fünfundsechzigsten Geburtstag, Mainz 1994

Kailitz, Susanne, Auseinandersetzungen mit der Gewalt - Frankfurter Schule, Studentenbewegung und RAF, in: Jahrbuch Extremismus \& Demokratie 16 (2004), S. 83-102

Karl, Michaela, Rudi Dutschke. Revolutionär ohne Revolution, Frankfurt a. M. 2003

Keitel, Ulrich, „Sehr geehrter Parteifreund...“. Parteiinterne Rundbriefe gegen alte Nazis, Frankfurt a. M. 2001

Kersting, Franz-Werner, „Unruhediskurs“. Zeitgenössische Deutungen der 68er-Bewegung, in: Frese/Paulus/Teppe, Demokratisierung und gesellschaftlicher Aufbruch, S. 715-740

Kersting, Franz-Werner, Entzauberung des Mythos? Ausgangsbedingungen und Tendenzen einer gesellschaftlichen Standortbestimmung der westdeutschen ,68 er'-Bewegung, in: Westfälische Forschungen 48 (1998), S.1-19

Kersting, Franz-Werner, Psychiatriereform und ,68`, in: Westfälische Forschungen 48 (1998), S. 283-295

Kielmansegg, Peter Graf, Nach der Katastrophe. Eine Geschichte des geteilten Deutschland, Berlin 2000

Kirchgässner, Hubert (Hg.), Texte zur soziokulturellen Animation, 2. veränd. Aufl., Remscheid 1983

Kirsch, Werner, Entscheidungsprozesse (3 Bde.), Wiesbaden 1970/71

Klein, Armin, Kulturpolitik. Eine Einführung, Opladen 2003

Kleßmann, Christoph, Zwei Staaten, eine Nation. Deutsche Geschichte 1955-1970, 2. überarb. u. erw. Aufl., Bonn 1997

Kleßmann, Christoph, 1968 - Studentenrevolte oder Kulturrevolution, in: Hettling, Manfred (Hg.), Revolution in Deutschland? 1789-1989, Göttingen 1991, S. 90-105

Knöpfle, Franziska, Im Zeichen der Soziokultur. Hermann Glaser und die kommunale Kulturpolitik in Nürnberg, Nürnberg 2007

Koenen, Gerd, Das rote Jahrzehnt. Unsere kleine deutsche Kulturrevolution 1967-1977, Köln 2001

Koenen, Gerd, Der transzendental Obdachlose - Hans-Jürgen Krahl, in: Zeitschrift für Ideengeschichte, Heft II/3, 2008, S. 5-22

Köhler, Gerd/Reuter, Ernst (Hg.), Was sollen Schüler lernen? Die Kontroverse um die hessischen Rahmenrichtlinien für die Unterrichtsfächer Deutsch und Gesellschaftslehre. Dokumentation einer Tagung der Gewerkschaft Erziehung und Wissenschaft, Frankfurt a.M. 1973

Köser, Helmut (Hg.), Der Bürger in der Gemeinde. Kommunalpolitik und politische Willensbildung, Bonn 1979

Kohl, Helmut, Erinnerungen 1982-1990, München 2005

Kolb, Eberhard, Die Weimarer Republik, 7. Aufl., München 2009

Kolb, Eberhard, Die Arbeiterräte in der deutschen Innenpolitik 1918 bis 1919, Düsseldorf 1962

Kommunale Kulturpolitik in Dokumenten: Berichte, Projekte, Konzepte (hg. vom Dt. Institut für Urbanistik), bearb. von Gabriele Mosbach und Albrecht Göschel, Berlin 1991

Kommunale Kulturpolitik: Thesen, hg. vom Institut für Kommunalwissenschaften, Sankt Augustin, 2. unveränd. Aufl., Sankt Augustin 1981 
Korff, Gottfried/Roth, Martin (Hg.), Das historische Museum. Labor, Schaubühne, Identitätsfabrik, Frankfurt a.M. u.a. 1990

Korte, Hermann, Eine Gesellschaft im Aufbruch. Die Bundesrepublik Deutschland in den sechziger Jahren, Frankfurt a. M. 1987

Koschnick, Hans (Hg.), Der Abschied vom Extremistenbeschluß, Bonn 1979

Koselleck, Reinhart, Vergangene Zukunft. Zur Semantik geschichtlicher Zeiten, 6. Aufl., Frankfurt a.M. 2006 (zuerst 1979)

Kötter, Kirsten, Der Frankfurter Kunstverein in den 70er Jahren (unveröffentlichte Magisterarbeit am Kunstgeschichtlichen Institut der Universität Frankfurt), Frankfurt a.M. 1992 (Bibliothek des IfSG)

Krabbe, Wolfgang R., Parteijugend in Deutschland. Junge Union, Jungsozialisten und Jungdemokraten 1945-1980, Wiesbaden 2002

Krahl, Hans-Jürgen, Konstitution und Klassenkampf. Schriften und Reden 1966-1970, 4. Aufl., Frankfurt a. M. 1985

Krahl, Hilde, Ich bin fast immer angekommen. Erinnerungen. Aufgezeichnet von Dieter H. Bratsch, 2. Aufl., München 1998

Kramer, Dieter, Handlungsfeld Kultur. Zwanzig Jahre Nachdenken über Kulturpolitik, Essen 1996

Kraus, Dorothea, Zwischen Selbst- und Mitbestimmung: Demokratisierungskonzepte im westdeutschen Theater der frühen siebziger Jahre, in: Gilcher-Holtey, Politisches Theater, S. $125-152$

Krause-Burger, Sibylle, Joschka Fischer. Der Marsch durch die Illusionen, Reinbek bei Hamburg 2000 (zuerst 1995)

Kraushaar, Wolfgang, 1968 als Mythos, Chiffre und Zäsur, Hamburg 2000

Kraushaar, Wolfgang, Der lange Marsch durch die Institutionen, in: Landgrebe/Plath (Hg.), '68 und die Folgen, S.61-64

Kraushaar, Wolfgang, 1968. Das Jahr, das alles verändert hat, München u.a. 1998

Kraushaar, Wolfgang, Die Protest-Chronik 1949-59. Eine illustrierte Geschichte von Bewegung, Widerstand und Utopie, Hamburg 1996

Kraushaar, Wolfgang (Hg.), Frankfurter Schule und Studentenbewegung. Von der Flaschenpost zum Molotowcocktail 1946-1995, 2. Aufl., Hamburg 1998, Bd.1-3; Bd.1: Chronik, Bd. 2: Dokumente, Bd.3: Kommentare und Aufsätze, Register

Kraushaar, Wolfgang, Fischer in Frankfurt, Hamburg 2001

Kraushaar, Wolfgang, Die Frankfurter Sponti-Szene. Eine Subkultur als politische Versuchsanordnung, in: Archiv für Sozialgeschichte 44 (2004), S. 105-121

Kraushaar, Wolfgang, Achtundsechzig. Eine Bilanz, Berlin 2008

Krauss, Marita, Nachkriegskultur in München. Münchner städtische Kulturpolitik 19451954, München 1985

Kreuder, Friedemann, Formen des Erinnerns im Theater Klaus Michael Grübers, Berlin 2002

Krohn, Maren, Die gesellschaftliche Auseinandersetzung um die Notstandsgesetze, Köln 1981

Kuhn, Annette/Schneider, Gerhard (Hg.), Geschichte lernen im Museum, Düsseldorf 1978

Kunst der bürgerlichen Revolution von 1830 bis 1848/49. Zusammengestellt und herausgegeben aus Anlaß der Ausstellung im Schloß Charlottenburg, Berlin 1972/73, 3. verb. Aufl., Berlin 1973

Kulturpolitik. Mit Beiträgen von Wolfgang Lipp u. a., Landeszentrale für politische Bildung Baden-Württemberg, Stuttgart/Berlin/Köln 1989

Kunstpädagogisches Zentrum im Germanischen Nationalmuseum (Hg.), Zum Beispiel Dürer-Studio. Dokumentation und Kritik eines ausstellungsdidaktischen Experiments von Jürgen Rohmeder u. a., Ravensburg 1972

Küpper, Jost, Die SPD und der Orientierungsrahmen '85, Bonn-Bad Godesberg 1977

Kuratorium Kulturelles Frankfurt (Hg.), Nur ein Kulturverein? Information, Rückblick, Bestandsaufnahme, Appell, Frankfurt a.M. 1978

Kurzenberger, Hajo, Theaterkollektive: Von der ,Truppe 31' zur ,Marthaler-Familie', von der Politisierung der 68er-Bewegung zur Privatisierung des Theatermachens in den Neunzigern, in: Gilcher-Holtey, Politisches Theater, S. 153-178 
Landgrebe, Christiane/Plath, Jörg (Hg.), '68 und die Folgen. Ein unvollständiges Lexikon, Berlin 1998

Lange, Wigand, Theater in Deutschland nach 1945. Zur Theaterpolitik der amerikanischen Besatzungsbehörden, Frankfurt a. M. u. a. 1980

Langguth, Gerd, Protestbewegung: Entwicklung. Niedergang. Renaissance. Die Neue Linke seit 1968, 2. Aufl., Köln 1984

Langguth, Gerd, Mythos '68. Die Gewaltphilosophie von Rudi Dutschke. Ursachen und Folgen der Studentenbewegung, München 2001

Laufner, Richard, Am Fuß der blauen Berge. Kulturpolitik, Stadtentwicklung und Alternativkultur am Beispiel Marburg, Marburg 1982

Lefebvre, Henri, Revolution der Städte, München 1972

Lemire, Laurent, Cohn-Bendit, Paris 1998

Lohmar, Ulrich, Demokratisierung in Deutschland. Vorträge u. Aufsätze, Gütersloh 1969

Lohmar, Ulrich, Innerparteiliche Demokratie. Eine Untersuchung der Verfassungswirklichkeit politischer Parteien in der Bundesrepublik Deutschland, Stuttgart 1968 (zuerst 1963)

Lönnendonker, Siegward (Hg.), Linksintellektueller Aufbruch zwischen „Kulturrevolution“ und „kultureller Zerstörung“. Der Sozialistische Deutsche Studentenbund (SDS) in der Nachkriegsgeschichte (1946-1969). Dokumentation eines Symposiums, Opladen 1998

Lösche, Peter/Walter, Franz, Die SPD. Klassenpartei - Volkspartei - Quotenpartei. Zur Entwicklung der Sozialdemokratie von Weimar bis zur deutschen Vereinigung, Darmstadt 1992

Loschütz, Gert, in Zusammenarbeit mit Horst Laube (Hg.), War da was? Theaterarbeit und Mitbestimmung am Schauspiel Frankfurt 1972-1980, Frankfurt a. M. 1980

Löwisch, Manfred/Kaiser, Dagmar, Tendenzschutz in öffentlich-rechtlich geführten Bühnenunternehmen. $\mathrm{Zu}$ den verfassungsrechtlichen Grenzen der Landespersonalvertretungsgesetze, Baden-Baden 1996

Lucke, Albrecht von, 68 oder neues Biedermeier. Der Kampf um die Deutungsmacht, Berlin 2008

Lübbe, Hermann, Der Mythos der ,kritischen Generation‘. Ein Rückblick, in: APuZ 20 (1988), S. 17-25

Lübbe, Hermann, 1968. Zur kulturellen und politischen Wirkungsgeschichte in Deutschland, in: Schubert, Venanz (Hg.), 1968. 30 Jahre danach, St. Ottilien 1999, S.185-208

Luft, Friedrich, Vorwort, in: 25 Jahre Theater in Berlin. Theaterpremieren 1945-1970 (hg. im Auftrag des Senats von Berlin), Berlin 1972, S.7-20

Machiavelli, Niccolò, Discorsi. Gedanken über Politik und Staatsführung, Dt. Gesamtausgabe, 3. verb. Aufl., Stuttgart 2007

Maier, Hans, 1968 - Versuch einer Bilanz, hg. vom Bayerischen Landtag (Akademiegespräch), München 2002

Marcuse, Herbert, Über den affirmativen Charakter der Kultur, in: Marcuse, Herbert, Kultur und Gesellschaft I, Frankfurt a.M. 1965

Marwick, Arthur, The sixties. Cultural Revolution in Britain, France, Italy and the United States, 1958-1974, Oxford u.a. 1998

Matthias, Erich, Zwischen Räten und Geheimräten. Die deutsche Revolutionsregierung 1918/19, Düsseldorf 1970

Melchinger, Siegfried, Geschichte des politischen Theaters, Hannover 1971

Mennicken, Rainer, Peter Palitzsch. Regie im Theater, Frankfurt a. M. 1993

Menzel, Hans-Jochen, Die Rechte des Betriebsrats im Tendenzbetrieb nach dem Betriebsverfassungsgesetz vom 15. Januar 1972, Diss. Regensburg 1978

Merkelbach, Valentin, Pressekampagne gegen das Konzept eines historischen Museums, in: Hoffmann/Junker/Schirmbeck, Geschichte als öffentliches Ärgernis, S. 259-266

Merseburger, Peter, Willy Brandt 1913-1992. Visionär und Realist, Stuttgart u. a. 2002

Mertz, Peter, Das gerettete Theater. Die deutsche Bühne im Wiederaufbau, Weinheim u.a. 1990

Miermeister, Jürgen, Rudi Dutschke. Mit Selbstzeugnissen und Bilddokumenten, 6. Aufl., Reinbek 2003 (zuerst 1986) 
Mitscherlich, Alexander, Die Unwirtlichkeit unserer Städte. Anstiftung zum Unfrieden, Frankfurt a.M. 1965

Mohr, Albert Richard, Das Frankfurter Opernhaus 1880-1980. Ein Beitrag zur Frankfurter Theatergeschichte, Frankfurt a. M. 1980

Mosler, Peter, Was wir wollten, was wir wurden. Studentenrevolte - zehn Jahre danach. Mit einer Chronologie von Wolfgang Kraushaar, Reinbek bei Hamburg 1977

Müller, André, Kreuzzug gegen Brecht. Die Kampagne in der Bundesrepublik 1961/62, Berlin 1962

Müller, Harald/Schitthelm, Jürgen (Hg.), 40 Jahre Schaubühne Berlin, Berlin 2002

Müller-Doohm, Stefan, Adorno. Eine Biographie, Frankfurt a. M. 2003

Müller-Rommel, Ferdinand, Innerparteiliche Gruppierungen in der SPD. Eine empirische Studie über informell-organisierte Gruppierungen in der SPD von 1969-1980, Opladen 1982

Mündemann, Tobias, Die 68er ... und was aus ihnen geworden ist, München 1988

Nagel, Ivan, Drama und Theater. Von Shakespeare bis Jelinek, München u. a. 2006

Negt, Oskar, Achtundsechzig. Politische Intellektuelle und die Macht, 4.Aufl., Göttingen 2008 (zuerst 1995)

Neuenfels, Hans, Erinnern und sich erinnern. Nachruf auf Peter Palitzsch, in: Iden, Peter Palitzsch, S. 181-194

Neuenfels, Hans, Gedichte. Ovar und Opium. 1. Absprung in anderen Atem, Wiesbaden 1960

Neuenfels, Hans, Mundmündig [Gedichte], Köln 1963

Niedenhoff, Horst-Udo, Auf dem Marsch durch die Institutionen. Die kommunistische Agitation im Betrieb und in den Gewerkschaften, Köln 1979

Niedenhoff, Horst-Udo, Mitbestimmung in der Bundesrepublik Deutschland, Köln 1979

Nipperdey, Thomas, Konflikt, einzige Wahrheit der Gesellschaft? Zur Kritik der hessischen Rahmenrichtlinien, Osnabrück 1974

Noll, Gerhard, Arbeitsrecht im Tendenzbetrieb. Ratgeber für Arbeitnehmer und deren Interessenvertretung, Frankfurt a.M. 2001

Oberpriller, Martin, Jungsozialisten. Parteijugend zwischen Anpassung und Opposition, Bonn 2004

Oelinger, Josef, Die neue Linke und der SDS. Die politische Theorie der revolutionären Opposition, Köln 1969

Oertzen, Peter von, Betriebsräte in der Novemberrevolution. Eine politikwissenschaftliche Untersuchung über Ideengehalt und Struktur der betrieblichen und wirtschaftlichen Arbeiterräte in der deutschen Revolution 1918/19, Düsseldorf 1963

Offe, Claus, Einleitung, in: Bachrach, Peter/Baratz, Morton S., Macht und Armut. Eine theoretisch-empirische Untersuchung, Frankfurt a. M. 1977, S.7-34.

Osswald, Albert, Eine Zeit vergeht. Lebenserinnerungen des ehemaligen hessischen Ministerpräsidenten, 2. Aufl., Gießen 1994

Otto, Karl A., Vom Ostermarsch zur APO. Geschichte der außerparlamentarischen Opposition in der Bundesrepublik Deutschland 1960-1970. Mit einem Nachwort von Andreas Buro, Frankfurt a. M. u. a. 1977

Otto, Karl A., APO. Außerparlamentarische Opposition in Quellen und Dokumenten (1960-1970), Köln 1989

Pauls, Hermann, Herkommen und Persönlichkeit, in: Von Verschuer, Nikolaus/Gres, Joachim (Hg.), Liber amicorum für Alexander Riesenkampff zum siebzigsten Geburtstag, München 2006, S. 261-269

Plagemann, Volker, Beitrag, in: Fünf Jahrzehnte kommunale Kulturpolitik, Köln 1992 (Deutscher Städtetag-Beiträge zur Bildungs- und Kulturpolitik, Heft 20)

Podevils, Clemens Graf, Tendenzwende. Zur geistigen Situation in der Bundesrepublik, Stuttgart 1975

Poeche, Sabine, Mitbestimmung in wissenschaftlichen Tendenzbetrieben, Baden-Baden 1999 
Pohlmann, Markus, Kulturpolitik in Deutschland. Städtisch organisierte Kultur und Kulturadministration, München 1994

Preiß, Achim/Stamm, Karl/Zehnder, Frank Günter (Hg.), Das Museum - Die Entwicklung in den 80er Jahren. Festschrift für Hugo Borger zum 65. Geburtstag, München 1990

Ramthun, Gudrun, Kulturpolitik in Krefeld nach dem 2. Weltkrieg, Frankfurt a.M./Bern 1982

Rauth, Oliver, Die hessische Schulpolitik nach dem Großen Hessenplan. Eine Politikfeldanalyse des Konfliktes um die Rahmenrichtlinien, Marburg 2001 (Diplomarbeit am FB Gesellschaftswissenschaften und Philosophie)

Rebentisch, Dieter, Ludwig Landmann. Frankfurter Oberbürgermeister der Weimarer Republik, Wiesbaden 1975

Reinicke, Helmut, Für Krahl, Berlin 1973

Reinhardt, Hermann, CDU und SPD - ein Vergleich ihrer kommunalen Kulturpolitik (unveröff. verwaltungswissenschaftliche Diplomarbeit der Universität Konstanz) 1981 (Bibliothek der CDU-Fraktion im Frankfurter Römer)

Reisner, Stefan (Hg.), Briefe an Rudi D. Eingeleitet von Rudi Dutschke, Frankfurt a.M. 1968 (Voltaire-Flugschriften, 19)

Richter, Pavel A., Die Außerparlamentarische Opposition in der Bundesrepublik Deutschland 1966 bis 1968, in: Gilcher-Holtey, 1968, S.35-55

Riedelsheimer, Heinz, Der Tendenzbetrieb in arbeitsrechtlicher und verfassungsrechtlicher Sicht, Diss. Erlangen 1958

Rischbieter, Henning (Hg.), Durch den eisernen Vorhang. Theater im geteilten Deutschland 1945 bis 1990, Berlin 1999

Rinsche, Günter, Die CDU in den Großstädten, in: Dietrich Rollmann (Hg.), Die Zukunft der CDU. Christlich-demokratische Konzeption für die Zukunft, Hamburg 1968, S. 192 210

Roberts, David (Hg.), Tendenzwenden. Aspekte des Kulturwandels der Siebziger Jahre, Frankfurt a. M. u. a. 1984

Rödder, Andreas, Die Bundesrepublik Deutschland 1969-1990, München 2004

Rödder, Andreas, Wertewandel und Postmoderne. Gesellschaft und Kultur der Bundesrepublik Deutschland 1965-1990, Stuttgart 2004

Röhl, Bettina, So macht Kommunismus Spaß! Ulrike Meinhof, Klaus-Rainer Röhl und die Akte Konkret, Hamburg 2006

Rohmeder, Jürgen, Das Dürer-Studio. Entstehung, Beschreibung, Betrieb und Echo, in: Zum Beispiel Dürer-Studio, S.12-70

Rohrmoser, Günter, Die Herausforderung der Radikalen. Zwölf Kolumnen zum Zeitgeschehen, Köln 1973

Rohstock, Anne, Von der „Ordinarienuniversität“ zur „Revolutionszentrale“? Hochschulreform und Hochschulrevolte in Bayern und Hessen 1957-1976, München 2010

Roik, Michael, Die DKP und die demokratischen Parteien 1968-1984, Paderborn u.a. 2006

Rotberg, Joachim, Zwischen Linkskatholizismus und bürgerlicher Sammlung. Die Anfänge der CDU in Frankfurt am Main 1945-1946, Frankfurt a.M. 1999

Rotberg, Joachim, Vom „Katholikenausschuß“ zur Volkspartei. Die CDU Frankfurt am Main 1945-2005, in: ders./Zimmer, Aus Liebe zu Frankfurt, S.17-58

Rotberg, Joachim/Zimmer, Matthias, Aus Liebe zu Frankfurt. Erinnerungen und Streiflichter aus 60 Jahren CDU, hg. vom CDU-Kreisverband Frankfurt am Main, Frankfurt a. M. 2005

Roth, Jürgen, Z. B. Frankfurt. Die Zerstörung einer Stadt, München 1975

Roth, Roland, Rebellische Subjektivität. Herbert Marcuse und die neuen Protestbewegungen, Frankfurt a.M. 1985

Ruck, Michael, Ein kurzer Sommer der konkreten Utopie - Zur westdeutschen Planungsgeschichte der langen 60er Jahre, in: Schildt/Siegfried/Lammers, Dynamische Zeiten, S.362401

Rudorf, Reginald, Die vierte Gewalt. Das linke Medienkartell, Frankfurt a. M./Berlin 1994 
Rudzio, Wolfgang, Eine Erneuerung gesellschaftsverändernder Kommunalpolitik, in: Naßmacher, Karl-Heinz (Hg.), Kommunalpolitik und Sozialdemokratie. Der Beitrag des demokratischen Sozialismus zur kommunalen Selbstverwaltung, Bonn-Bad Godesberg 1977, S. 78-110

Rühle, Günther, Anarchie in der Regie, Frankfurt a. M. 1982

Rühle, Günther, Die Wiederherstellung des Theaters, in: Hoffmann/Klotz, Die Kultur, Bd. 5, S. 73-89

Rühle, Otto, Illustrierte Kultur- und Sittengeschichte des Proletariats, Bd.1, Berlin 1930 (Nachdruck Lahn-Gießen 1977)

Rürup, Reinhard, Probleme der Revolution in Deutschland 1918/19, Wiesbaden 1968

Rüschenschmidt, Heinrich, Gründung und Anfänge der CDU in Hessen, Darmstadt 1981

Schauer, Helmut (Hg.), Prima Klima. Wider den Zeitgeist. 1. Gnadenlose Generaldebatte zur endgültigen Klärung aller unzeitgemäßen Fragen, Hamburg 1987

Schelsky, Helmut, Die Strategie der „Systemüberwindung“. Der lange Marsch durch die Institutionen, in: ders., Systemüberwindung, Demokratisierung und Gewaltenteilung. Grundsatzkonflikte der Bundesrepublik, 4. unveränd. Auflage, München 1974, S. 19-37

Schelsky, Helmut, Die skeptische Generation. Eine Generation der deutschen Jugend. Mit einem Vorwort zur Taschenbuchausgabe, ungekürzte Ausgabe, Frankfurt 1975 (zuerst 1957)

Scheytt, Oliver, Rechtsgrundlagen der kommunalen Kulturarbeit, Köln 1994

Schieb, Roswitha, Peter Stein. Ein Porträt, Berlin 2005

Schildt, Axel/Siegfried, Detlef/Lammers, Karl Christian (Hg.), Dynamische Zeiten. Die 60er Jahre in den beiden deutschen Gesellschaften, 2. Aufl., Hamburg 2003 (zuerst 2000)

Schildt, Axel, Materieller Wohlstand - pragmatische Politik - kulturelle Umbrüche. Die 60er Jahre in der Bundesrepublik, in: ders./Siegfried/Lammers, Dynamische Zeiten, S. 2153

Schildt, Axel, „Die Kräfte der Gegenreform sind auf breiter Front angetreten“. Zur konservativen Tendenzwende in den Siebzigerjahren, in: Archiv für Sozialgeschichte 44 (2004), S. 449-478

Schirmbeck, Peter, Zur Museumsdidaktik, in: Hoffmann/Junker/Schirmbeck, Geschichte als öffentliches Ärgernis, S. 283-297

Schlak, Stephan, Wilhelm Hennis. Szenen einer Ideengeschichte der Bundesrepublik, München 2008

Schmidtke, Michael, Der Aufbruch der jungen Intelligenz. Die 68er Jahre in der Bundesrepublik und den USA, Frankfurt a. M. 2003

Schmökel, Reinhard/Kaiser, Bruno, Die vergessene Regierung. Die große Koalition 1966 bis 1969 und ihre langfristigen Wirkungen, Bonn 1991

Schneider, Franz (Hg.), Dienstjubiläum einer Revolte. „1968“ und 25 Jahre, München 1993

Schneider, Michael, Demokratie in Gefahr? Der Konflikt um die Notstandsgesetze. Sozialdemokratie, Gewerkschaften und intellektueller Protest, 1958-1968, Bonn 1986

Schneider, Dieter/Neuland, Franz/Möller, Walter, Zwischen Römer und Revolution, 18691969. Hundert Jahre Sozialdemokraten in Frankfurt am Main, Frankfurt a. M. 1969

Schomann, Bernd, Frankfurter Portraits. Eine Stadt und ihre Menschen, Rödermark 1995

Schonauer, Karlheinz, Die ungeliebten Kinder der Mutter SPD. Die Geschichte der Jusos von der braven Parteijugend zur innerparteilichen Opposition, Bonn 1982

Schönberger, Irmingard (Hg.), Betrachten + bewahren. Ein Querschnitt durch das schriftliche Werk von Arno Schönberger, Nürnberg 1995

Schönbohm, Wulf, Die CDU wird moderne Volkspartei. Selbstverständnis, Mitglieder, Organisation und Apparat 1950-1980, Stuttgart 1985

Schöndienst, Eugen, Geschichte des Deutschen Bühnenvereins seit 1945. Ein Beitrag zur Geschichte des Theaters, Frankfurt a.M. 1981

Schönhoven, Klaus, Wendejahre. Die Sozialdemokratie in der Zeit der Großen Koalition 1966-1969, Bonn 2004

Schuchardt, Helga (Hg.), Kultur im Alltag. Neue Formen kommunaler Kulturpolitik, Hamburg 1985 
Schulz, Kristina, Der lange Atem der Provokation. Die Frauenbewegung in der Bundesrepublik und in Frankreich 1968-1976, Frankfurt a. M. 2002

Schulz, Kristina, Studentische Bewegungen und Protestkampagnen, in: Roth, Roland/ Rucht, Dieter, Die sozialen Bewegungen in Deutschland seit 1945. Ein Handbuch, Frankfurt a.M. u. a. 2008, S. 417-446

Schulze, Gerhard, Die Erlebnisgesellschaft. Kultursoziologie der Gegenwart, Frankfurt a.M. /New York 1992

Schütte, Wolfram (Hg.), Adorno in Frankfurt. Ein Kaleidoskop mit Texten und Bildern, Frankfurt a.M. 2003

Schwarz, Elisabeth, Ein Mann und (s)ein Modell. Peter Palitzsch und die Mitbestimmung, in: Mennicken, Peter Palitzsch, S. 78-86

Schwarz, Hans-Peter, Die Ära Adenauer. Gründerjahre der Republik 1949-1957, Stuttgart 1981

Schwencke, Olaf/Sievers, Norbert, Kulturpolitik ist Gesellschaftspolitik. Festschrift zum siebzigsten Geburtstag von Alfons Spielhoff, 2. erw. und aktual. Ausgabe, Hagen 1988

Schwencke, Olaf, Kulturpolitik ist Gesellschaftspolitik, in: ders./Sievers, Kulturpolitik ist Gesellschaftspolitik, S.1-8

Schwencke, Olaf/Revermann, Klaus H./Spielhoff, Alfons (Hg.), Plädoyers für eine neue Kulturpolitik, München 1974

Schweppenhäuser, Gerhard, Theodor W. Adorno zur Einführung, 3. überarb. Aufl., Hamburg 2003

Schwiedrzik, Wolfgang Matthias, Theater als „Aktion“, in: Gilcher-Holtey, Vom Ereignis zum Gegenstand, S. 224-238

Schwier, Hans, Jede Epoche schafft ihre Museen, in: Preiß/Stamm/Zehnder (Hg.), Das Museum - Die Entwicklung in den 80er Jahren, S.77-80

Seeling, August, Leitsätze zur kommunalen Kulturarbeit, in: Grimme, Adolf (Hg.), Kulturverwaltung der zwanziger Jahre, Stuttgart 1961, S. 115-120

Seier, Hellmut, Paul Kluke 1908-1990. Nekrolog, in: HZ 252 (1991), S. 212-215

Siepmann, Eckhard, Che-Schah-Shit. Die sechziger Jahre zwischen Cocktail und Molotow, Reinbek bei Hamburg 1988

Soell, Hartmut, Helmut Schmidt: 1918-1969. Vernunft und Leidenschaft, München 2003

Sozialistischer Lehrerbund (Hg.), Materialien zur Schulbuchproduktion. Analysen, Tendenzen, Alternativen, Offenbach 1973 (Fotodr. ULB Darmstadt)

Soukup, Uwe, Wie starb Benno Ohnesorg? Der 2. Juni 1967, Berlin 2007

SPD-Unterbezirk Frankfurt am Main, Mehr Demokratie wagen. Der Fall Littmann. Eine Dokumentation, Frankfurt a. M. 1970

Spickernagel, Ellen/Walbe, Brigitte (Hg.), Das Museum: Lernort contra Musentempel, 2. Aufl., Gießen 1976 (Sonderband der Zeitschrift „Kritische Berichte“)

Städtische Kulturpolitik. Empfehlungen, Richtlinien und Hinweise des Deutschen Städtetages zur Praxis städtischer Kulturpolitik 1946-1970. Neue Schriften des Deutschen Städtetages, Köln 1971

Stahl, Karl Heinz, Georg Lucász und die Zerstörung der Vernunft. Eine Würdigung des Goethepreisträgers 1970, in: Tribüne 9 (1970), 35, S.3808-3816

Stamer, Sabine, Cohn-Bendit. Die Biografie, Hamburg 2001

Stecklina, Gerd/Schille, Joachim (Hg.), Otto Rühle. Leben und Werk (1874-1943), Weinheim/München 2003

Stehen, Jürgen, Didaktische Aspekte einer Theorie des Historischen Museums, in: Kuhn/ Schneider, Geschichte lernen im Museum, S. 49-81

Steinmetz, Willibald, Von der Geschichte der Gesellschaft zur „Neuen Kulturgeschichte“, in: Wirsching, Andreas (Hg.); Neueste Zeit, München 2006, S. 233-250

Stephan, Dieter, Jungsozialisten: Stabilisierung nach langer Krise? Theorie und Politik 19691979. Eine Bilanz, Bonn 1979

Stern, Klaus/Hermann, Jörg, Andreas Baader. Das Leben eines Staatsfeindes, 2. Aufl., München 2007

Stettner, Herbert, Parteifest, Frankfurt a. M. 1991 
Stracke, Ernst, Stadtzerstörung und Stadtteilkampf. Innerstädtische Umstrukturierungsprozesse, Wohnungsnot und soziale Bewegungen in Frankfurt am Main, Köln 1980

Streeck, Sylvia u. Wolfgang, Parteiensystem und Status quo. Drei Studien zum innerparteilichen Konflikt, Frankfurt a. M. 1972

Stubenvoll, Hans/Schirmbeck, Peter, Dokumentation 20. Jahrhundert, in: Hoffmann/Junker/Schirmbeck, Geschichte als öffentliches Ärgernis, S. 153-218

Sucher, Curt Bernd (Hg.), Theaterlexikon. Autoren, Regisseure, Schauspieler, Dramaturgen, Bühnenbildner, Kritiker, München 1995

Sucher, Curt Bernd, Luc Bondy. Erfinder, Spieler, Liebhaber, Salzburg u. a. 2002

Süß, Dietmar, Die Enkel auf den Barrikaden. Jungsozialisten in der SPD in den Siebzigerjahren, in: Archiv für Sozialgeschichte 44 (2004), S.67-104

Thalheimer, August, Einführung in den dialektischen Materialismus (Die moderne Weltanschauung), Wien/Berlin 1928 (Nachdruck Bremen 1973)

Thränhardt, Dietrich, Geschichte der Bundesrepublik Deutschland, Frankfurt a. M. 1996

Timm, Uwe, Heißer Sommer, Köln 1985

Tödtmann, Hermann-Ulrich, Die Zulässigkeit tarifvertraglicher Mitbestimmungsregelungen für Bühnenkünstler, Diss. Köln 1995

Töteberg, Michael, Rainer Werner Fassbinder, Reinbek bei Hamburg 2002

Uhle, Reinhard, Pädagogik der siebziger Jahre. Zwischen wissenschaftsorientierter Bildung und repressionsarmer Erziehung, in: Faulstich, Werner (Hg.), Die Kultur der 70er Jahre, München 2004, S. 49-64

Urban, Peter (zusammengestellt von), Das Buch vom Verlag der Autoren 1969-1989. Beschreibung eines Modells und seiner Entwicklung, Frankfurt a. M. 1989

Vilmar, Fritz, Strategien der Demokratisierung, Bd.1: Theorie der Praxis; Bd.2: Modelle und Kämpfe der Praxis, Darmstadt/Neuwied 1973

Vogel, Hans-Jochen, Die Amtskette. Meine zwölf Münchner Jahre. Ein Erlebnisbericht, München 1972

Waetzoldt, Stephan, Das Museum als Quelle der Information, in: Bott, Das Museum, S. 279284

Waldmann, Sabine, „Es muß alles anders werden, wurscht was!“ Die Entwicklung politischen Denkens und Handelns bei ehemaligen APO-Studenten, München/Wien 1991

Wallmann, Walter, Zur strafrechtlichen Problematik des Züchtigungsrechtes der Lehrer. Unter besonderer Berücksichtigung des geltenden Verfassungsrechtes, Diss. Marburg 1965

Wallmann, Walter, Der Preis des Fortschritts. Beiträge zur politischen Kultur, Stuttgart 1983

Wallmann, Walter, Im Lichte der Paulskirche. Memoiren eines Politischen, Potsdam 2002

Walter, Franz, Die Achtundsechziger. Liberale Zäsur der Republik?, in: Universitas 53 (1998), S. 957-961

Walter, Franz, Die SPD. Vom Proletariat zur Neuen Mitte, Berlin 2002

Was bleibt. Walter Möller in seinen Aufsätzen, Reden, Zitaten, Interviews. Hg. vom Presseund Informationsamt der Stadt Frankfurt am Main, Frankfurt a. M. 1972

Weber, Max, Wirtschaft und Gesellschaft, Tübingen 1922

Weber, Petra, Carlo Schmid. 1896-1979. Eine Biographie, München 1996

Wehler, Hans-Ulrich, Bismarck und der Imperialismus, Köln 1969

Wehling, Hans-Georg/Lipp, Wolfgang, Kulturpolitik, Stuttgart u. a. 1989

Weidhaas, Peter, Zur Geschichte der Frankfurter Buchmesse, Frankfurt a. M. 2003

Weiss, Heipe, Fuchstanz, Frankfurt a. M. 1999

Wekwerth, Manfred, Erinnern ist Leben. Eine dramatische Autobiographie, Leipzig 2000

Wesel, Uwe, Die verspielte Revolution. 1968 und die Folgen, München 2002

Wiedemeyer, Wolfgang, Gefragt: Walter Wallmann, Bornheim 1977

Wiggershaus, Rolf, Die Frankfurter Schule. Geschichte, theoretische Entwicklung, politische Bedeutung, 6. Aufl., München 2001 (zuerst 1986) 
Willeke, Rudolf, Die geistigen Hintergründe der Kulturrevolution 1968-1998 und ihre Folgen für Familie und Kirche, Gesellschaft und Staat, hg. von der Komturei Münster/Westfalen des Ordo Militiae Crucis Templi, Tempelherren-Orden Deutsches Priorat, Münster 2000

Wirsching, Andreas, Abschied vom Provisorium 1982-1990, München 2006

Wolf, Werner, Neubeginn und Kampf um die Mehrheit. Die CDU Hessen unter Alfred Dregger 1967-1982, in: Wolf, Werner (Hg.), CDU Hessen 1945-1985. Politische Mitgestaltung und Kampf um die Mehrheit, Köln 1986, S. 59-97

Wolff, Karl D. (Hg.), 5x7 Lesebuch: 1970-2005. 35 Jahre Stroemfeld/Roter Stern, Frankfurt a.M. 2005

Wolfrum, Edgar, Die geglückte Demokratie. Geschichte der Bundesrepublik Deutschland von ihren Anfängen bis zur Gegenwart, Stuttgart 2006

Zehetmair, Hans, Kultur bewegt. Kulturpolitik für Bayern, München 2001

Zellmer, Elisabeth, Töchter der Revolte? Frauenbewegung und Feminismus in den 1970er Jahren in München, München 2011

10 Jahre Ernst-Reuter-Schule: Gesamtschule in der Nordweststadt Frankfurt am Main. Eine Zwischenbilanz, hg. von der Kollegialen Schulleitung der Ernst-Reuter-Schule I u. II., Redaktion: Herbert Stubenrauch, Frankfurt a. M. 1974 



\section{Abkürzungsverzeichnis}

$\begin{array}{ll}\text { ACDP } & \text { Archiv für Christlich-Demokratische Politik } \\ \text { AdsD } & \text { Archiv der sozialen Demokratie } \\ \text { AG } & \text { Aktiengesellschaft } \\ \text { APO } & \text { Außerparlamentarische Opposition } \\ \text { AStA } & \text { Allgemeiner Studentenausschuss } \\ \text { BV } & \text { Bezirksverband } \\ \text { CDU } & \text { Christlich-Demokratische Partei Deutschlands } \\ \text { CSU } & \text { Christlich-Soziale Union in Bayern } \\ \text { CSSR } & \text { Tschechoslowakische Sozialistische Republik } \\ \text { DDR } & \text { Deutsche Demokratische Republik } \\ \text { DGB } & \text { Deutscher Gewerkschaftsbund } \\ \text { DM } & \text { Deutsche Mark } \\ \text { DKP } & \text { Deutsche Kommunistische Partei } \\ \text { DST } & \text { Deutscher Städtetag } \\ \text { EWG } & \text { Europäische Wirtschaftsgemeinschaft } \\ \text { FAZ } & \text { Frankfurter Allgemeine Zeitung } \\ \text { FBfV } & \text { Frankfurter Bund für Volksbildung } \\ \text { FDP } & \text { Freie Demokratische Partei Deutschlands } \\ \text { FDJ } & \text { Freie Deutsche Jugend } \\ \text { Ffm } & \text { Frankfurt am Main } \\ \text { FNP } & \text { Frankfurter Neue Presse } \\ \text { FR } & \text { Frankfurter Rundschau } \\ \text { GDBA } & \text { Genossenschaft Deutscher Bühnen-Angehöriger } \\ \text { HiMu } & \text { Historisches Museum } \\ \text { HKB } & \text { Höchster Kreisblatt } \\ \text { HR } & \text { Hessischer Rundfunk } \\ \text { IfSG } & \text { Institut für Stadtgeschichte (der Stadt Frankfurt am Main) } \\ \text { IfZ } & \text { Institut für Zeitgeschichte } \\ \text { IG } & \text { Industriegewerkschaft } \\ \text { IHK } & \text { Industrie- und Handelskammer } \\ \text { JU } & \text { Junge Union (Jugendorganisation von CDU und CSU) } \\ \text { Judos } & \text { Jungdemokraten (FDP-naher Jugendverband) } \\ \text { Jusos } & \text { Arbeitsgemeinschaft der Jungsozialisten in der SPD } \\ \text { KB } & \text { Künstlerischer Beirat } \\ \text { KBW } & \text { Kommunistischer Bund Westdeutschland } \\ \text { KMK } & \text { Kultusministerkonferenz } \\ \text { KPD } & \text { Kommunistische Partei Deutschlands } \\ \text { KPV } & \text { Kommunalpolitische Vereinigung (der CDU/CSU) } \\ \text { KV } & \text { Kreisverband } \\ & \end{array}$


KZ Konzentrationslager

LA Landesarchiv

MSP(D) Mehrheitssozialdemokratische Partei (Deutschlands)

NDR Norddeutscher Rundfunk

NPD Nationaldemokratische Partei Deutschlands

Nr. Nummer

$\mathrm{OB} \quad$ Oberbürgermeister

PDS Partei des Demokratischen Sozialismus

RAF Rote Armee Fraktion

SB Städtische Bühnen

SEW Sozialistische Einheitspartei Westberlin

SDAJ Sozialistische Deutsche Arbeiterjugend

SDS Sozialistischer Deutscher Studentenbund

SPD Sozialdemokratische Partei Deutschlands

SS Schutzstaffel

SZ Süddeutsche Zeitung

Stvv Stadtverordnetenversammlung

TAT Theater am Turm

TOP Tagesordnungspunkt

UB Unterbezirk

Ufa Universum Film AG

UNESCO United Nations Educational, Scientific and Cultural Organization

USP(D) Unabhängige Sozialdemokratische Partei (Deutschlands)

USA United States of America

VHS Volkshochschule

ZDF Zweites Deutsches Fernsehen 


\section{Personenregister}

Abela, Albert 377,378

Abendroth, Wolfgang 15, 151, 395

Abs, Hermann Josef 113, 114, 396-398

Adenauer, Konrad 36, 54, 80, 91, 93

Adlhoch, Walter 142, 143, 430

Adorno, Theodor W. 15, 29, 42, 43, 49, 50, 104, 308

Adrian, Hans 360, 369, 391

Agnoli, Johannes 16

Ahrlé, Ferry 345

Albertz, Luise 105

Alvarez, Santiago, 117

Amann, Max 215

Ambrosius, Gerhard 89-91

Andernacht, Dietrich 139

Appel, Rudolf $334,336,370$

Arndt, Rudi 2, 13, 23, 25, 61, 67, 96, 115, $128,131,136,140,147,281,312,316$, $323,327,328,330,332-335,343,344$, 347-351, 354-367, 369-375, 377-379, 381, 386-389, 399, 401, 405, 410, 411, 414-416, 418-420, 422-425, 427, 431, 432, 434-439, 441, 442, 448

Arold, Antje 363

Arroyo, Eduardo 243

Assmann, Arno 180, 181

Augstein, Rudolf 7

Baader, Andreas 49

Bachmann, Karl 331

Balser, Frolinde $3,67,101-103,107,136$, 146, 158, 199, 410, 424

Barfuß, Grischa 173, 174

Barth, Susanne 275

Bartsch, Wolfgang 86, 135, 148

Barzel, Rainer $105,276,332,343,348$, 426

Bauer, Friedrich-Wilhelm 159, 401, 403, 409

Baumert, Wolfgang 367, 370, 387

Baumgart, Reinhard 311

Beaucamp, Eduard 397, 400

Bebel, August 146

Becker, Karl 78, 79, 90

Beckett, Samuel Barclay 312

Beckmann, Ruth 87, 94

Bender, Kurt 90, 337
Benedict, Jörg 196

Benn, Gottfried 447

Benneter, Klaus-Uwe 435

Berg, Martin 75, 332, 356, 359-361, 376, 426, 432

Berg, Werner 183

Berkemeier, Karlheinz $\quad 63,369,436$

Bermbach, Udo 75

Bernauer, Ernst 87

Bernhard, Patrick 12

Bethmann, Johann Philipp Freiherr von 55, 86-88, 95, 128, 129, 134, 331, 383

Bethmann, Moritz Freiherr von 86

Beuys, Joseph 393, 448

Biedenkopf, Kurt 426

Bingel, Horst 401

Bismarck, Otto von 160-163, 408, 449

Bloch, Ernst 104, 121, 131, 275

Bloomfield, Theodore 176

Bock, Karl-Albert 229

Bockelmann, Werner 28

Bodisco, Dirk von 235, 275, 276, 286

Böhlke, Edgar M. 222, 255, 281, 288, 316

Böll, Heinrich 105

Börner, Holger 370, 430, 434

Bösch, Frank 54

Bond, Edward 220, 234, 245, 246, 271, 303-307, 311

Bondy, Luc 242, 243, 246, 247, 255, 258, 261, 262, 268, 286-289, 311, 312, 316

Borger, Hugo 165

Born, Horst 65, 66

Bott, Gerhard 126

Bracker, Jörgen 165-167

Brakert, Gisela 202

Brandt, Willy 9, $13,15,55,58,60,66,67$, $71,73,105,133,148,327,333,348,368$, $372,388,442,443,447$

Braun, Karlheinz 296-301, 454

Brecht, Bertolt $31,32,38,47,171,173$, 177, 178, 182, 187, 190, 201, 220-224, 226, 230, 238, 244, 246, 265, 269, 275, $302,308,309,311,316,317,451,452$, 456

Brecht, Ulrich 39

Breithaupt, Dankwart 361

Breithaupt, Anita $326,355,362,370$ 
Brooks, Peter 201

Brundert, Willi 23, 37, 39, 45, 46, 48, 60, 63, 65, 67, 70-72, 79, 173-175, 196-198, 204, 325, 329, 367, 438, 442, 452

Bubis, Ignatz 51

Buckwitz, Harry 5, 30-32, 36, 38, 39, 46, 111, 171, 172, 174, 177, 179, 180, 249, 314,451

Bude, Heinz 241

Büchner, Georg 227

Buhre, Traugott $229,259,277,291,304$, 316

Burg, Lou van 407

Burgholz, Richard 64, 66

Buselmeier, Michael 191

Bussmann, Georg 393-395, 401

Canaris, Volker 321

Castagne, Helmut 174, 177, 179

Castro, Fidel 117

Chopin, Frédéric François 447

Clauss, Armin 356

Cocteau, Jean 311

Cohen, Max 158

Cohn-Bendit, Daniel 9, 23, 49, 51, 372, 436, 459

Conradt, Gerd 117

Conze, Werner 102

Corterier, Peter 57

Däubler-Gmelin, Herta 128

Däumig, Ernst 158

Dahrendorf, Ralf 7

Danzeisen, Peter 235, 237-240, 249, 250, 253, 257, 271, 274, 278, 279, 281-286, 293, 298, 316, 444

Debray, Régis 6

Deichsel, Wolfgang 120, 392

Deppisch, Hans-Werner 181

Diederich, Reiner 394

Diehl, Siegfried 392, 401

Diekhoff, Marlen 229, 244, 247, 256, 262, 286

Dietz, Fritz $\quad 88,114,398,399$

Dietz, Hans 83, 89, 91

Dirks, Walter 86

Döll, Klaus 81, 94, 331, 338, 350, 380, 381, 383

Dohnányi, Christoph von 176, 193, 195, 399

Dorst, Tankred 224, 230

Dregger, Alfred $1,6,16,24,73,77,79,80$, $86-88,92,140,288,336,337,347,348,384$, 412, 415, 416, 419, 426, 432, 437, 439, 447

Drese, Claus Helmut 191, 192

Dürer, Albrecht 126, 127
Dürrenmatt, Friedrich 98

Düttmann, Johannes 107, 409

Dutschke, Rudi 6-8, 16, 44-46, 50, 62, 63, $148,185,189,339,445,447$

Ebert, Friedrich 122, 147, 155-157

Echternach, Jürgen 332

Eckert, Rainer 326, 334, 335, 355, 356, $362,365,444$

Ehlers, Joachim 141, 146

Ehre, Ida 177

Ehrenberg, Herbert 61,378

Ehrlich, Wilfried 359,360

Eichel, Hans 135

Eichhorn, Werner 196

Ellwein, Thomas 12

Elste, Wilfried 282

Engelhardt, Ernst Günther 220

Engelmann, Bernt 162

Engelmann, Ingeborg 229, 258

Engels, Friedrich 275

Ensslin, Gudrun 49

Erfurth, Ulrich $5,111,172,174-180,192-$ 206, 211, 212, 215, 451, 452

Erhard, Ludwig 36, 54, 105

Ernst, Max 226

Eschenburg, Theodor 69

Everding, August 189, 197, 201, 211, 217, 451

Faller, Herbert $\quad 70,74,75,325$

Faßbender, Heinrich 352

Fassbinder, Rainer Werner 5, 108, 314, 392, 393

Fay, Wilhelm 24, 78, 79, 174, 175, 209, $328,329,331,334,336,337,339,341$, $344,347,348,350,353,380-383,395$, 401,413

Feik, Eberhard $\quad 245,250,252,256,257$, 261, 267, 268

Fernandes, Augusto 236, 256

Fetscher, Iring 345

Feydeau, Georges 194

Fichter, Tilmann 12

Fieg, Wolfgang 326

Filz, Günter 424

Fischer, Fritz 153

Fischer, Joseph (Joschka) 9, 19, 23, 49, 51,459

Fischer, Jürgen $\quad 234,261,275,278,286$

Flach, Karl Hermann 76

Flesch-Thebesius, Max 96

Flesch-Thebesius, Marlies 144

Fletcher, Lucille 290

Flimm, Jürgen 321

Fogt, Helmut $\quad 446,447$ 
Frank, Anne 136

Franke, Peter 239, 247, 248, 289, 291, 292

Freiwald, Friedrich $\quad 85,86,94$

Freyh, Brigitte 62

Friedeburg, Ludwig von $14,50,131,169$, $365,373,433$

Friedrich, Erhard 184, 298

Friedrich, Rudolf 389

Frisch, Max 47

Fritzsche, Klaus 373

Frobenius, Leo 97

Früh, Giovanni 229

Fuchs, Matthias 240, 260, 301

Furth, Peter 322

Gainsborough, Thomas 447

Gandhi, Mohandas Karamchand 359

Gall, Lothar 114, 168, 414

Gallwitz, Klaus 397, 398

Gansel, Norbert 57

Garnier, Hans-Detlev von $81-84,89,93$, $331,338,350,380,383,385$

Gauland, Alexander 2, 398, 448

Gavajda, Peter (Pedro) 275

Gebhardt, Fred 61, 101, 118, 326, 331, $333,340,357,359,363-372,374,375$, $378,424,426,430,436,437,442,444$

Gehlen, Arnold 6

Geiss, Imanuel 153-157, 160-162, 164

Geißler, Emil 330

Gelhaar, Klaus 215, 225, 227, 231-237, $240,246,260,261,263,264,283,286$, 304,305

Genet, Jean 31

Genscher, Hans-Dietrich 433

Gerhardt, Ernst $78,81-83,85,87-94,115$, $116,329,335-337,376,382,383,388$, $413,429,430,437,457$

Gilcher-Holtey, Ingrid 22, 167, 188, 456

Gillessen, Günther 133, 141, 143, 149

Gladkow, Fjodor 247

Glaser, Hermann 12, 21, 33-35, 103, 314

Goebbels, Joseph 142, 176, 347

Goethe, Wolfgang von 31, 40, 193, 318

Görtemaker, Manfred 19

Göttig, Willy Werner 172

Gorki, Maxim 310

Gottmann, Günther 165

Grass, Günter 105, 202, 203, 205

Grebing, Helga 147

Grüber, Klaus Michael 222, 224, 243, 309, 316

Grübling, Richard 394

Gründgens, Gustaf 174, 177, 196, 277

Günther, Herbert 373
Guevara, Ernesto Che 6

Guillaume, Christel 66,70

Guillaume, Günter 67

Gustafson, Lars 214

Haad, Ulrich 229

Habermas, Jürgen 44

Hacks, Peter 193

Hagenau, Heinz 120, 243, 249

Hamm-Brücher, Hildegard 105

Handke, Peter 120, 183, 200, 315

Hankel, Wilhelm 345

Hartmann, Rainer $\quad 160,177,178,193,312$, 315

Haug, Winfried 346

Haverkampf, Hans-Erhard 369

Hebbel, Friedrich 311

Heck, Bruno 15, 54, 92, 95

Heckroth, Hein 196

Heiber, Helmut 154

Heidegger, Martin 104

Hein, Bastian 455

Heinemann, Gustav 105

Heinrichs, Benjamin 304, 305, 313

Henrichs, Helmut 243

Held, Hans Ludwig 33

Helmholz, Bodo 349

Hellwig, Hans-Jürgen $\quad 81,380,383$

Hennis, Wilhelm 75

Hensel, Andreas 269, 292

Hensel, Georg 203, 294, 295

Hermand, Jost 21

Hesselbach, Walter 330

Hessler, Hermann 172

Hetzer, Willi 47, 48

Heumann, Jochem, 84, 91

Heym, Heinrich 29, 129, 130, 306, 307

Heyme, Hansgünther 187

Hilpert, Heinz 179, 181

Hilpert, Werner 86

Hochhuth, Rolf 38, 105, 187

Hodenberg, Christina von 20, 445

Höcherl, Hermann 105

Hoffmann, Alexander 334

Hoffmann, Detlef $130,133,166,168,406$, 444,450

Hoffmann, Hilmar 4, 5, 9, 12-14, 22-24, $103-119,121,122,130,131,134,136$, $137,146,147,149,150,160,163,164$, 167, 205-215, 227, 241, 243-245, 251, $270,271,281,285,289,296,298,302$, $303,306,307,310,312,317,320,323$, $329,340,341,344,345,351,391-407$, 409, 410, 422, 447, 448, 450-452, 456, 457

Hoffmann, Detlef 139, 143, 153, 154, 162, 450 
Hohenemser, Herbert $\quad 40,125,202,217$, 218

Holz, Hans Heinz 121, 122

Holzamer, Johannes Karl 40

Holzapfel, Hartmut 135, 395

Horn, Wolfgang 22

Horkheimer, Max 42, 43, 50

Hübner, Kurt 173, 174, 223

Huonder Guido 268, 277, 310

Ibsen, Henrik Johan 310,317

Iden, Peter 172, 193, 194, 200, 207, 210, 222, 224, 226, 234, 240-242, 276, 282, 290, 296, 297, 300, 305, 314-317

Ignée, Wolfgang 243

Ivo, Hubert 134, 450

Jacobi, Ernst $\quad 244,248,267,269,272,274-$ 276, 452

Jäkel, Peter $325,357,360,370,375$

Jäger, Gerd 316

Janßen, Karl-Heinz $\quad 135,149,151$

Jeker, Valentin 243, 245, 260, 268, 277

Johler, Jens $182,184,185,189,190$

John, Gottfried 256

Johnson, Lyndon B. 224

Kabelitz, Roland 201

Kämpf, Günter 137

Kanther, Manfred 412

Karajan, Herbert von 227

Karasek, Hellmuth 207, 313-315

Karry, Heinz-Herbert $128,210,349,350$, 352, 389

Keaton, Buster 341

Keitel, Ulrich $150,159,163$

Kentrup, Norbert 262, 263, 265, 291

Khuon, Ulrich 313

Kiesinger, Kurt Georg 54, 79, 85

King, Martin Luther 359

Kipphoff, Petra 165, 168

Kirchgäßner, Alfons 38

Kirchner, Gottfried 150

Kiskalt, Hans $45,63,64,72,324,339$

Klaußnitzer, Horst 185-187

Kleinschmidt, Peter 192, 193, 196-200

Kleist, Armin 363, 365, 366, 369

Klett, Ernst 13

Klett, Renate 244, 266

Kloth, Jürgen $239,253,258,275,279$, 283-289

Kluge, Alexander 105

Kluke, Paul 142, 154-157

Kneidl, Karl 215

Knobel, Enno 151

Knoeringen, Waldemar von 33
Koch, Heinrich 31, 196

Koch, Rainer 167

Köhler, Franz-Heinz 308

Koenen, Gerd 445

Koenigs, Tom 459

Köper, Carmen-Renate 47

Köpke, Horst 302, 305, 400

Kohl, Helmut 1, 54, 385, 411, 412, 415, 416, 426, 437, 439

Kolb, Eberhard 156, 438

Kollwitz, Käthe 394

Korenke, Hans Ulrich 29, 109, 110, 199, 200, 211, 214, 318, 340, 341, 346, 391, 393, 408

Korn, Karl 132, 172

Kortner, Fritz 300

Koselleck, Reinhart 17

Krämer-Badoni, Rudolf $\quad 101,113,117-$ 119, 149, 203, 204, 216, 311, 315, 317, 407, 448, 449

Krahl, Hans-Jürgen $\quad 48,49$

Krahl, Hilde 177

Kraus Dorothea 22, 188

Kraushaar, Wolfgang 2, 6

Kreling, Josef 343

Krollmann, Hans 373

Lafontaine, Oscar 443

Lampel, Peter Martin 246

Landmann, Ludwig 136

Lang, Joseph $70,330,356,358$

Langguth, Gerd 439

Laube, Heinrich 181

Laube, Horst $22,207,231,233,240-243$, 246, 247, 261, 263, 264, 268, 269, 273, $275,276,278,281,282,285,286,290-$ 293, 295, 297, 299, 311, 315-317, 456

Leber, Georg $\quad 60-62,70,74,335,356,388$

Leggewie, Claus 19

Leimbacher, Christoph 286

Leisler-Kiep, Walther 411, 416

Liebknecht, Karl 147

Lietzau, Hans 184, 185

Lindeiner-Waldau, Klaus von $\quad 352,390$

Lingnau, Hermann 114, 115

Link, Helmut 87, 93, 341

Lissner, Erich 172,177

Littmann, Gerhard 45, 68-73, 75, 76, 103, 107, 339, 438

Lösche, Peter 21, 147

Löscher, Peter 301, 312

Lorca, Federico García 311

Lorenz, Konrad 48

Lorenz, Peter 387

Loschütz, Gert 22, 265

Lucácz, Georg 339 
Lübbe, Hermann 455

Lütgert, Gert 72

Luft, Friedrich 31

Luxemburg, Rosa 122

Macourek, Miloš 194

Mahler, Horst 56

Maier, Hans 459

Mann, Golo 414

Mann, Thomas 105, 338

Mao Zedong 6, 292

Marcuse, Herbert $10,34,35,126,188$, 449

Marx, Karl 43, 62, 144, 149, 275, 328, 394

Matthöfer, Hans 62

Mehring, Franz 156

Meinhof, Ulrike 427

Mende, Erich 105

Mennicken, Rainer 321

Merkelbach, Valentin 134, 450

Michel, Hans 346, 357, 371, 376, 416, 418, 420

Mick, Günter 367, 376

Millowitsch, Willy 176

Minden, Heinrich $120,212,243,246,285$, 286

Minks, Wilfried 206, 210, 215, 231, 320

Mischnick, Wolfgang 350

Mitscherlich, Alexander 27, 35, 342

Möller, Alex 153

Möller, Walter 13, 23, 28, 60-77, 79, 80, $84,98,99,101,103,106,107,115,204$, 205, 324-330, 349, 367, 398, 441, 442, 447, 452

Mörger, Ursula 196

Molnar, Franz (Ferenc Molnár) 290

Monk, Egon 173, 174, 308

Moog, Hans-Jürgen $78,80,81,96,97$, $121,122,348,350,354,380,402$, 407, 413, 431

Müller, André 189, 201

Müller, Felix 120

Müller, Hans-Reinhardt 174

Müller, Heiner 247, 313

Müller, Knut 421

Münch, Richard 196, 197, 199, 200, 202, 203, 206

Mündemann, Tobias 12

Neander, Joachim 85

Negt, Oskar 87

Neuenfels, Hans 206, 215, 220, 225-231, 233, 236, 237, 240, 242, 243, 245, 246, 252, $253,258,260,268,271,275-282,285$, 286, 288-291, 307, 309-311, 313-317, 453

Neuss, Wolfgang 189
Niemetz, Alexander 80

Nitzling, Erich 370,374

Noelte, Rudolf 211, 222

Noske, Gustav 155

Oertzen, Peter von $\quad 61,156,363$

Oertzen, Tania von 225, 256, 257

Ohnesorg, Benno 44

Orth, Elsbeth 141, 146

Osswald, Albert 72, 330, 367, 379, 386, 387, 389, 422, 434

Otting, Ludwig von 321

Palitzsch, Peter $5,173,181,206,207,209$ 216, 219-226, 228-231, 233-237, 239-260, 267-269, 272, 273, 275-290, 292-297, 299-307, 309-315, 317, 318, 320, 321, 341, 404, 409, 452, 453, 455-457

Pappermann, Ernst 19

Papst Paul VI. 143

Peichl, Markus 446

Petri, Roland 118, 119, 121, 392

Peymann, Claus 120, 173, 192, 193, 197, 198, 200, 207, 234

Picht, Georg 128, 446

Pinter, Harold 193, 316

Piscator, Erwin 31, 187

Pleß, Philipp 330

Polzer, Erhard 362

Postel, Helmut 310

Potthoff, Heinrich 147

Raabe, Christian $\quad 63,65,199,326,330$, 355-357, 444

Raabe, Wilhelm 245, 327

Rabehl, Bernd 7, 185, 445

Rabenau, Burghard von 352

Radke, Olaf 61

Raspe, Jan-Carl 154

Rath, Karl vom 28, 29, 101, 196, 199, 200, 203, 328, 452

Rauhe, Gernot 206

Rebentisch, Dieter W. 136, 141, 146, 157

Reber, Horst 137, 138, 167

Redl, Christian 255, 261

Reible, Dieter 47, 192-200, 207

Reiss, Willi 325, 327, 376

Rethel, Alfred 133

Retschy, Gerhard 120, 249, 274, 285, 294

Reuter, Wolfgang 86

Revermann, Klaus H. 22, 308

Rhein, Peter 343, 423, 432

Riemer, Horst 78

Rischbieter, Henning $\quad$ 184, 207, 270, 309

Riesenhuber, Heinz 87, 382-389, 413, 416, $418,429,437,438,457$ 
Riesenkampff, Alexander 81, 85, 331, 341, 350, 383, 385, 386, 397, 428

Robespierre, Maximilien de 48

Rökk, Marika 176

Roggisch, Peter 225, 229, 236, 246, 254, 256, 257, 260, 274, 283, 284, 287, 292, $301,318,345$

Rohde, Gerhard 317

Rohmeder, Jürgen 127

Rohstock, Anne 20

Rosenberg, Arthur 145, 155

Rotberg, Joachim 25

Roth, Jürgen 378, 421

Rudel, Hans-Ulrich 406

Rudolph, Dieter 390

Rudolph, Hans-Christian 301

Rudolph, Niels-Peter 215

Rudzio, Wolfgang 25, 65, 69, 70, 76, 325, 332, 444

Rueffer, Michael Alwin 212, 213

Rühle, Günther 171, 172, 176, 207, 295, 303-305, 315, 316, 319, 320

Rühle, Otto $132,140,142,144,156,167$, 449

Rühmann, Heinz 176

Rürup, Reinhard 156

Sabeis, Heinz-Winfried 308

Sackenheim, Friedrich Franz 99, 101, 103, 107, 199, 200, 203, 298, 330, 410

Sartre, Jean-Paul 31

Sauberzweig, Dieter 4, 41, 166

Schaaf, Johannes 320

Scharnagel, Karl 33

Schauer, Helmut 11

Schauss, Hans Joachim 120

Scheel, Walter 351

Scheidemann, Philipp 122, 147

Schelsky, Helmut 20, 446, 447

Schiller, Friedrich 308

Schirmbeck, Peter 145

Schmahl, Hildegard 256

Schmid, Carlo 33, 44

Schmidt, Doris 168

Schmidt, Helmut $58,368,426,435,436$, 437, 442, 443

Schmidtke, Michael 20, 445

Schmitt-Vockenhausen, Hermann $\quad 2,74$

Schnitzler, Eduard von 117

Schoeler, Andreas von $352,376,389,390$, 416, 431, 433

Schönberger, Arno 166

Schönberger, Hans 381

Schopohl, Eos 320

Schreiner, Otmar 435

Schrenck-Notzing, Caspar von 14
Schubart, Alexander $347,357,359,360$, $365,370,371,378,404,436,444$

Schuh, Oscar Fritz 174, 180, 308

Schumacher, Kurt 347

Schwab, Ulrich $243,249,250,290$

Schwarz, Elisabeth 228, 229, 235, 237, $246,253,254,261,262,265,281,283$, $285,287,318$

Schwarz, Sonja 178

Schwarz-Schilling, Christian 411

Schwarzenstein, Christa Mette Mumm von 381

Schwencke, Olaf 22

Schwiedrzik, Wolfgang Matthias 23, 189, 190, 451

Schwing, Hugo 245

Seghers, Anna 223

Seib-Art, Walther 148

Seidel, Hans-Dieter $304,305,317$

Seifert-Mitscherlich, Monika 343

Seiters, Rudolf 77

Sellner, Gustav Rudolf 31

Senghor, Leopold 49

Seybold, Eberhard $\quad 219,317$

Shakespeare, William 48, 177, 193, 303

Sichtermann, Barbara 182, 184, 185, 188, 190

Siegfried, Detlef 20

Sieglerschmidt, Hellmut 324

Siepmann, Eckard 22

Sigulla, Adalbert 67,70

Soares, Mário 372

Sölch, Rudi 356-358, 361, 364, 369, 376, 442

Sollwedel, Inge $390,418,433$

Solti, Georg 30, 399

Spielhoff, Alfons 4, 22

Sprengel, Bernhard 400

Springer, Axel Cäsar 45

Stanislawski, Konstantin Sergejewitsch 222

Steckel, Frank-Patrick 311

Stefanek, Lore 229, 259, 261-263, 273, 291, 301

Steiger, Klaus 225, 259, 276, 285

Stein, Corinna 258

Stein, Peter $120,173,189,190,192,193$, 198-200, 207, 212, 307, 451

Steiof, Adolf Christian 231, 233, 234, 236, 237, 240, 262, 266, 286, 295, 297

Sternheim, Carl 31

Stettner, Herbert $128,129,130,176,177$

Stettner, Wilhelm 40

Stock, Wolfgang Jean 133

Storey, David 310

Stratenwerth, Christoph 287 
Strauß, Botho 318

Strauß, Franz Josef $87,238,332,338,343$, $348,415,416,424,431$

Streeck, Sylvia 21, 76

Streeck, Wolfgang 21, 76, 326

Strien, Eva-Maria 239, 248

Strindberg, August 392

Stubenvoll, Hans 127, 130, 132-134, 137, $139,141,145,147,148,150,157-159$, 161,167

Sturmfels, Klaus 436

Suhrkamp, Peter 296

Sukowa, Barbara $254,255,259,260,262$, 263, 266, 318

Sulimma, Burkhard 405

Svevo, Italo 248

Terson, Peter 226

Thalheimer, August 277

Thomazewski, Otto 421

Tillian, Robert 255

Timm, Uwe 171

Toller, Ernst 224

Trageser, Karl-Heinz 407

Trakl, Wolfgang 7

Trampert, Rainer 10

Traube, Klaus 435

Treusch, Hermann $\quad 240,244,276,286$, 288, 404, 406

Trissenaar, Elisabeth $\quad 228-230,239,251,318$

Tschechow, Anton Pawlowitsch 248, 320

Tucholsky, Kurt 47, 394

Ulbricht, Lutz 345

Ulbricht, Walter, 16, 74, 223

Ulrich, Sylvia 214, 233, 252, 274, 279

Ulrich, Volker 153

Unseld, Siegfried 113

Uslar, Jochen von 169

Uttendörfer, Walter 261, 267

Utzerath, Hansjörg $\quad$ 185, 190

Vequel-Westernach, Max von 225, 260, 263, 264

Viering, Stefan 265

Vilmar, Fritz 15, 16, 441

Völker, Klaus 215, 304

Vogel, Hans-Jochen 83, 105, 326, 367, 368

Voigt, Günther 149

Voigt, Karsten $\quad 25,43,44,58-61,68,70$, $74,87,118,330-333,340,357,361,362$, 364-371, 374, 421, 422, 444

Voitel, Gottfried 349-352

Vorbeck, Dorothee 76, 363, 370, 374

Voss, Reinhard $335,342,372,386$

Vring, Thomas von der 447
Waetzoldt, Stephan 125,126

Wallmann, Walter $1,18,23,24,84,89,92$, 94, 317, 387, 403, 404, 411-420, 429, 431, 432-434, 437-439, 448, 457

Walter, Franz 21

Warnke, Christof 144

Warren, Robert Penn 32

Weber, Hermann 122

Weck, Gerhard 64-66, 327

Wedekind, Frank 255, 311, 317

Wehler, Hans-Ulrich 162-164

Wehmeier, Jörg 220, 267, 268

Wehner, Herbert $71,115,420,431$

Weil, Felix 97

Weimar, Peter 144

Weiss, Peter $32,187,188$

Weisser, Jens 253, 268, 275

Weizsäcker, Carl Friedrich von 104

Weizsäcker, Richard von 426

Wekwerth, Manfred 220, 221, 223

Wendland, Jens 311, 317

Wendt, Ernst 184, 185

Wennemann, Klaus 239, 240, 253, 275, 286-288, 292, 299

Wentz, Martin 373, 421, 435, 436, 444

Weser, Rudi 373

Wiebel, Martin 185,190

Wiens, Wolfgang 120,392

Wilder, Thornton 31

Wilhelm I., Deutscher Kaiser und König von Preußen 38

Wirth, Willi 121

Wittgenstein, Casimir Prinz 85

Wöll, Hans 356, 377

Wörner, Manfred 406

Wolf, Armin 138139

Wolf, Hartmut 134

Wolf, Horst 435

Wolff, Karl-Dietrich (alias KD) 49, 154

Wolfrum, Edgar 19

Wonder, Erich 235, 288, 317

Wüllner, Heinrich 186, 217

Zadek, Peter $173,187,224,307$

Zander, Fred $\quad 60,74-76,324,325-327,330$, $331,333-335,337,346,354-358,361$, $362,364,423,442$

Zeis, Christian 97, 135, 159, 161, 163, 164, 390, 391, 401, 408, 409, 420, 433

Ziegler, Gerhard 377

Zink, Otto 86

Zinn, Georg August 151, 434

Zoglmann, Siegfried 352

Zwerenz, Gerhard 345 